\title{
Primes of height one and a class of Noetherian finitely presented algebras*
}

\author{
Isabel Goffa ${ }^{\dagger} \quad$ Eric Jespers Jan Okniński
}

\begin{abstract}
Constructions are given of Noetherian maximal orders that are finitely presented algebras over a field $K$, defined by monomial relations. In order to do this, it is shown that the underlying homogeneous information determines the algebraic structure of the algebra. So, it is natural to consider such algebras as semigroup algebras $K[S]$ and to investigate the structure of the monoid $S$. The relationship between the prime ideals of the algebra and those of the monoid $S$ is one of the main tools. Results analogous to fundamental facts known for the prime spectrum of algebras graded by a finite group are obtained. This is then applied to characterize a large class of prime Noetherian maximal orders that satisfy a polynomial identity, based on a special class of submonoids of polycyclic-by-finite groups. The main results are illustrated with new constructions of concrete classes of finitely presented algebras of this type.
\end{abstract}

Because of the role of Noetherian algebras in the algebraic approach in noncommutative geometry, new concrete classes of finitely presented algebras recently gained a lot of interest. Via the applications to the solutions of the YangBaxter equation, one such class also widens the interest into other fields, such as mathematical physics. These algebras are finitely generated, say by elements $x_{1}, \ldots, x_{n}$, and they have a presentation defined by $\left(\begin{array}{l}n \\ 2\end{array}\right)$ monomial relations of the form $x_{i} x_{j}=x_{k} x_{l}$ so that every word $x_{i} x_{j}$ appears at most once in a relation. Such algebras have been extensively studied, for example in 11, 12, 14, 20, 33. It was shown that they have a rich algebraic structure that resembles that of a polynomial algebra in finitely many commuting generators; in particular, they are prime Noetherian maximal orders. Clearly, these algebras can be considered as semigroup algebras $K[S]$, where $S$ is a monoid defined via a presentation as that of the algebra. It turns out that these algebras are closely related to group algebras since $S$ is a submonoid of a torsion-free abelian-by-finite group.

\footnotetext{
${ }^{*}$ Research partially supported by the Onderzoeksraad of Vrije Universiteit Brussel, Fonds voor Wetenschappelijk Onderzoek (Flanders), Flemish-Polish bilateral agreement BIL2005/VUB/06 and a MNiSW research grant N201 004 32/0088 (Poland).

${ }^{\dagger}$ Research funded by a Ph.D grant of the Institute for the Promotion of Innovation through Science and Technology in Flanders (IWT-Vlaanderen).

2000 Mathematics Subject Classification. Primary 16P40, 16H05, 16S36; Secondary 20M25, $16 \mathrm{~S} 15$.
} 
In this paper we further explore new constructions of Noetherian maximal orders that are finitely presented algebras which are defined via monomial relations. In other words, we look for new constructions of semigroup algebras $K[S]$ of this type. In general, it remain unsolved problems to characterize when an arbitrary semigroup algebra is Noetherian and when it is a prime Noetherian maximal order. Recall that the former question even for group algebras has been unresolved. The only class of groups for which a positive answer has been given is that of the polycyclic-by-finite groups. Hence it is natural to consider the problems first for semigroup algebras $K[S]$ of submonoids $S$ of polycyclicby-finite groups. A structural characterization of such algebras $K[S]$ that are right Noetherian was obtained by the authors in [22, 23]. Namely, $S$ has a group of quotients $G=S S^{-1}$ in which there is a normal subgroup $H$ of finite index so that $[H, H] \subseteq S$ and $S \cap H$ is finitely generated, or equivalently, $S$ has a group of quotients $G$ that contains normal subgroups $F$ and $N$ so that $F \subseteq N$, $F$ is a subgroup of finite index in the unit group $\mathrm{U}(S)$ of $S$, the group $N / F$ is abelian, $G / N$ is finite and $S \cap N$ is finitely generated. It follows that $K[S]$ is right Noetherian if and only if $K[S]$ is left Noetherian. We simply say that $K[S]$ is Noetherian. Also, if $S$ is a submonoid of a finitely generated group $G$ that has an abelian subgroup $A$ of finite index, then $K[S]$ is Noetherian if and only if $S \cap A$ is finitely generated. Recall that if $K[S]$ is Noetherian then $S$ is finitely generated and $K\left[S \cap G_{1}\right]$ is Noetherian for every subgroup $G_{1}$ of $G$. If, furthermore, $G_{1}$ is of finite index in $G$ then $K[S]$ is a finitely generated right (and left) $K\left[S \cap G_{1}\right]$-module. Due to a result of Chin and Quinn [8] on rings graded by polycyclic-by-finite groups, $K[S]$ is Noetherian if and only if $S$ satisfies the ascending chain condition on right (equivalently, left) ideals.

Concerning the second problem. If $S$ is an abelian monoid, Anderson [1, 2] proved that $K[S]$ is a prime Noetherian maximal order if and only if $S$ is a submonoid of a finitely generated torsion free abelian group, $S$ satisfies the ascending chain condition on ideals and $S$ is a maximal order in its group of quotients. More generally, Chouinard [9] proved that a commutative monoid algebra $K[S]$ is a Krull domain if and only if $S$ is a submonoid of a torsion free abelian group which satisfies the ascending chain condition on cyclic subgroups and $S$ is a Krull order in its group of quotients. In particular, it turns out that the height one prime ideals of $K[S]$ determined by the minimal primes of $S$ are crucial. Brown characterized group algebras $K[G]$ of a polycyclic-by-finite group $G$ that are Noetherian prime maximal orders [3, 4]. This turns out to be always the case if $G$ is a finitely generated torsion free abelian-by-finite group (equivalently, $K[G]$ is a Noetherian domain that satisfies a polynomial identity, PI for short). In this situation all height one primes are principally generated by a normal element. So, in the terminology of Chatters and Jordan [7, $K[G]$ is a unique factorization ring. The authors described in 21] when a semigroup algebra of a submonoid $S$ of a finitely generated abelian-by-finite group is a Noetherian maximal order that is a domain. The description is fully in terms of the monoid $S$. Examples of such monoids are the binomial monoids and more general monoids of $I$-type. As mentioned earlier in the introduction, the latter were introduced by Gateva-Ivanova and Van den Bergh in [14] and studied in 
several papers (see for example [11, 12, 15, 20, 33]) and generalized to monoids of $I G$-type in $[18$.

In this paper we continue the investigations of prime Noetherian maximal orders $K[S]$ for submonoids $S$ of a polycyclic-by-finite group $G=S S^{-1}$. Of course, knowledge of prime ideals is fundamental. The primes not intersecting $S$ come from primes in the group ring $K\left[S S^{-1}\right]$. These are rather well understood through the work of Roseblade (see for example [31]), and in particular, the height one primes can be handled via Brown's result. The crucial point in our investigations are thus the primes of $K[S]$ intersecting $S$ non-trivially. In case $K[S]$ is Noetherian and $G=S S^{-1}$ is torsion free, the following information was proved in [22].

1. If $Q$ is a prime ideal of $S$ then $K[Q]$ is a prime ideal of $K[S]$.

2. The height one prime ideals of $K[S]$ intersecting $S$ non-trivially are precisely the ideals $K[Q]$ with $Q$ a minimal prime ideal of $S$.

Note that $G$ being torsion free is equivalent with $K[G]$ being a domain (see $[32$, Theorem 37.5]).

In the first part of this paper we continue the study of prime ideals in case $K[S]$ is prime (and thus $G$ is not necessarily torsion free). We show that the first property does not remain valid, however the second part on primes of height one still remains true. As a consequence, we establish going up and going down properties between prime ideals of $S$ and prime ideals of $S \cap H$, where $H$ is a subgroup of finite index in $G$. These are the analogs of the important results (see [32, Theorem 17.9]) known on the prime ideal behaviour between a ring graded by a finite group and its homogeneous component of degree $e$ (the identity of the grading group). As an application it is shown that the classical Krull dimension $\operatorname{clKdim}(K[S])$ of $K[S]$ is the sum of the prime dimension of $S$ and the plinth length of the unit group $\mathrm{U}(S)$. Also, a result of Schelter is extended to the monoid $S$ : the prime dimension of $S$ is the sum of the height and depth of any prime ideal of $S$.

The information obtained on primes of height one then allows us in the second section to determine when a semigroup algebra $K[S]$ is a prime Noetherian maximal order provided that $G=S S^{-1}$ is a finitely generated abelian-by-finite group (that is, $K[S]$ satisfies a polynomial identity). The result reduces the problem to the structure of the monoid $S$ (in particular $S$ has to be a maximal order within its group of quotients $G$ ) and to that of $G$. The characterization obtained generalizes the one given in 21 in case $G$ is torsion free and it shows that the action of $G$ on minimal primes of some abelian submonoid of $S$ is very important (as was also discovered for the special case where $S$ is a finitely generated monoid of $I G$-type in [18). Finally, in the last section, we prove a useful criterion for verifying the maximal order property of such $S$. We then show how this, together with our main result, can be used to build new examples of finitely presented algebras $R$ (defined by monomial relations) that are maximal orders. Since the main result of the paper deals with semigroup algebras $K[S]$ of submonoids $S$ of groups, we first have to check that $R$ is defined by such 
submonoids. So, in particular, in the third section we show how to go from the language of presentations of algebras to the language of monoids and their semigroup algebras.

\section{Prime spectrum}

Let $K$ be a field. Recall that if $S$ is a monoid with a group of quotients $G=S S^{-1}$ then the semigroup algebra $K[S]$ is prime if and only if $K[G]$ is prime (28, Theorem 7.19]), or equivalently $G$ does not contain nontrivial finite normal subgroups. The latter can also be stated as $\Delta^{+}(G)=\{1\}$, where $\Delta^{+}(G)$ is the characteristic subgroup of $G$ consisting of the periodic elements with finitely many conjugates (see [32, Theorem 5.5]). By $\Delta(G)$ one denotes the subgroup of $G$ consisting of the elements with finitely many conjugates.

Also recall that an equivalence relation $\rho$ on a semigroup $S$ is said to be a

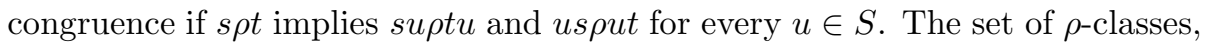
denoted $S / \rho$, has a natural semigroup structure inherited from $S$. In case $S$ has a group of quotients $G$ and $H$ is a normal subgroup of $G$ we denote by $\sim_{H}$ the congruence on $S$ defined by: $s \sim_{H} t$ if and only if $s=h t$ for some $h \in H$. If $G$ is a polycyclic-by-finite group then by $\mathrm{h}(G)$ we mean the Hirsch rank of $G$.

We often will make use of the following fact ([29, Lemma 3.1]). If $S$ is a submonoid of a finitely generated abelian-by-finite group $G$ then $S$ has a group of quotients $S \mathrm{Z}(S)^{-1}=\left\{s z^{-1} \mid s \in S, z \in \mathrm{Z}(S)\right\}$. It follows that if $T$ is a submonoid of a group $G$ that contains a normal subgroup $F$ so that $F \subseteq T$ and $G / F$ is finitely generated and abelian-by-finite (for example $T \subseteq G, G$ is polycyclic-by-finite and $T$ satisfies the ascending chain condition on right ideals, see [23]) then $T$ has a group of quotients that is obtained by inverting the elements $u$ of $T$ that are central modulo $F$. In particular, every (nonempty) ideal of $T$ contains such an element $u$.

Theorem 1.1 Let $S$ be a submonoid of a polycyclic-by-finite group, say with a group of quotients $G$, and let $K$ be a field. Assume that $S$ satisfies the ascending chain condition on right ideals and $G$ does not contain nontrivial finite normal subgroups. Then the height one prime ideals $P$ of $K[S]$ such that $P \cap S \neq \emptyset$ are exactly the ideals of the form $P=K[Q]$, where $Q$ is a minimal prime ideal of $S$.

Proof. Let $P$ be a height one prime ideal of $K[S]$ such that $Q=S \cap P \neq \emptyset$. By [30] (or see [24, Theorem 4.5.2], and also [24, Corollary 4.5.7]), $S / Q$ embeds into $\mathcal{M}_{n}(H)$, the semigroup of $n \times n$ monomial matrices over a group $H=T T^{-1}$ for a subsemigroup $T$ of $S$ such that $T \cap Q=\emptyset$. Furthermore, $K[S] / K[Q]$ embeds in the matrix algebra $M_{n}(K[H])$ and the latter is a localization of $K[S] / K[Q]$ with respect to an Ore set that does not intersect $P / K[Q]$. Subsemigroups of $S$ not intersecting $Q$ can be identified with subsemigroups of $\mathcal{M}_{n}(H)$. It is also known that there exists an ideal $I$ of $S$ containing $Q$ such that the nonzero elements of $I / Q$ are the matrices in $S / Q \subseteq \mathcal{M}_{n}(H)$ with exactly one nonzero entry. Furthermore, $T$ may be chosen so that $T \subseteq I \backslash Q$ and actually $T$ may be 
identified with $\left(e_{11} \mathcal{M}_{n}(H) e_{11} \cap(S / Q)\right) \backslash\{0\}$, where $e_{11}$ is a diagonal idempotent of rank one in $\mathcal{M}_{n}(H)$. Clearly $I / Q$ is an essential ideal in $S / Q$. We know that $S$ contains a normal subgroup $F$ of $G$ such that $G / F$ is abelian-by-finite. Then $H \subseteq F H$ and clearly $F H \cap Q=\emptyset$ and $F H=F T(F T)^{-1}$. Clearly $F T T F \subseteq$ $F H F=F H$, whence $(F T)(T F) \cap Q=\emptyset$. Since $T$ is a diagonal component of $I$ and $F T, T F \subseteq I$, by the matrix pattern on the matrices of rank one in $\mathcal{M}_{n}(H)$ it follows that $T F=F T=T$. Hence $H=\operatorname{gr}(T)=\operatorname{gr}(T F)=H F$.

Notice that we have a natural homomorphism $\phi: K[S] / K[Q] \longrightarrow K[S] / P$. Since $K[S]$ is Noetherian and as $M_{n}(K[H])$ is a localization of $K[S] / K[Q]$ with respect to an Ore set of regular elements that does not intersect $P / K[Q]$, there exists a prime ideal $R$ in $K[H]$ such that $M_{n}(K[H] / R)$ is a localization of $K[S] / P$, see [17, Theorem 9.22]. Moreover, since $P / K[Q]$ is a prime ideal of $K[S] / K[Q]$, we also have that $M_{n}(K[H] / R) \subseteq M_{n}\left(M_{t}(D)\right)$ for a division ring $D$ and a positive integer $t$ such that $M_{t}(D)$ is the ring of quotients of $K[H] / R$ and $\phi$ extends to a homomorphism $\phi^{\prime}: M_{n}(K[H]) \longrightarrow M_{n}(K[H] / R)$. Let $\rho_{P}$ be the congruence on $S$ defined by the condition: $(x, y) \in \rho_{P}$ if $x-y \in P$. Since the image of a nonzero entry of a matrix $s \in \mathcal{M}_{n}(H)$ under $\phi^{\prime}$ is invertible in $K[H] / R$, the rank of the matrix $\phi^{\prime}(s)$ is equal to $t$ multiplied by the number of nonzero entries of $s$. Thus, the ideal $J$ of matrices of rank at most $t$ in $S / \rho_{P} \subseteq$ $M_{n t}(D)$ satisfies $\phi^{-1}(J)=I$. Moreover, $S / \rho_{P}=\phi^{\prime}(S / Q) \subseteq \mathcal{M}_{n}\left(G L_{t}(D)\right)$ inherits the monomial pattern of $S / Q \subseteq \mathcal{M}_{n}(H)$. From [22, Corollary 4.4] we know that there is a right Ore subsemigroup $U$ of $I$ such that $\mathrm{h}\left(U U^{-1}\right)=$ $\mathrm{h}(G)-1$ and $U \cap P=\emptyset$, and also the image $U^{\prime}$ of $U$ in $S / \rho_{P}$ is contained in a diagonal component of $J$ (viewed as a monomial semigroup over $G L_{t}(D)$ ). Then $U$ can be identified with a subsemigroup of $S / Q$ and it is contained in a diagonal component of $S / Q$. It thus follows that $\mathrm{h}(H) \geq \mathrm{h}(\operatorname{gr}(U))=\mathrm{h}(G)-1$.

By the remark before the theorem we know that every ideal of $S$ contains an element of $S$ that is central modulo $F$. So, choose $z \in Q$ such that $z x \in x z F$ for every $x \in S$. Suppose that $z^{m} \in H$ for some positive integer $m$. Then $z^{m}=a b^{-1}$ for some $a, b \in T$. Hence $z^{m} b \in T \cap P$. As $T \cap P=\emptyset$, we obtain a contradiction. It thus follows that $\mathrm{h}(\operatorname{gr}(z, H))>\mathrm{h}(H) \geq \mathrm{h}(G)-1$. Therefore $\mathrm{h}(\operatorname{gr}(z, H))=\mathrm{h}(G)$ and, because $G$ is polycyclic-by-finite, we get $[G: \operatorname{gr}(z, H)]<\infty$. Since $z s \in s z F$ for every $s \in S \cap H$, it follows that $z(S \cap H) \subseteq(S \cap H) z F=(S \cap H F) z=(S \cap H) z$. As $H=(S \cap H)(S \cap H)^{-1}$, it follows that $z H=H z$. Therefore $\Delta^{+}(H)$ has finitely many conjugates in $G$. Since $\Delta^{+}(H)$ is finite, we get that $\Delta^{+}(H) \subseteq \Delta^{+}(G)=\{1\}$ and thus $K[H]$ is a prime algebra. Hence, the localization $M_{n}(K[H])$ of $K[S] / K[Q]$ is prime. So $K[Q]$ is a prime ideal in $K[S]$. As $K[S]$ is prime and $P$ is of height one, we get that $P=K[Q]$, as desired.

To prove the converse, let $Q$ be a minimal prime ideal of $S$. Let $P$ be an ideal of $K[S]$ maximal with respect to $S \cap P=Q$. Clearly $P$ is a prime ideal of $K[S]$. Again by the remark before the theorem, we know that there exists an element $z$ in $S$ that belongs to $Q$ and that is central modulo $F$. Then $z S=S z$, so $z$ is a normal element of $K[S]$. Since, by assumption $K[S]$ is a prime Noetherian algebra, the principal ideal theorem therefore yields a prime ideal $P^{\prime}$ of $K[S]$ that is of height one so that $z \in P^{\prime}$ and $P^{\prime} \subseteq P$. By the first part of the result, 
$P^{\prime}=K\left[S \cap P^{\prime}\right]$. Since $S \cap P^{\prime}$ is a prime ideal of $S$ contained in the minimal prime ideal $S \cap P=Q$, we get that $S \cap P^{\prime}=Q$. So $K[Q]=P^{\prime}$ is a height one prime ideal of $K[S]$.

Recall that the $\operatorname{rank} \operatorname{rk}(S)$ of a monoid $S$ (not necessarily cancellative) is the supremum of the ranks of the free abelian subsemigroups of $S$. The dimension $\operatorname{dim}(S)$ of $S$ is defined in the following way. By definition $\operatorname{dim} S=0$ if $S=\{e\}$. If $S$ has no zero element, then $\operatorname{dim}(S)$ is the maximal length $n$ of a chain $Q_{0} \subset Q_{1} \subset Q_{2} \subset \cdots \subset Q_{n}$, where $Q_{0}=\emptyset$ and $Q_{i}$ are prime ideals of $S$ for $i>0$ (note that primes are by definition different from $S$ ), or $\infty$ if such $n$ does not exist. If $\{e\} \neq S$ has a zero element, then $\operatorname{dim}(S)$ is the maximal length $n$ of such a chain with all $Q_{i}(i \geq 0)$ prime ideals of $S$, or $\infty$ if such $n$ does not exist. The spectrum $\operatorname{Spec}(S)$ is the set of all prime ideals of $S$.

We give an easy example that shows that Theorem 1.1 can not be extended to semigroup algebras that are not Noetherian. Let $S=\left\{x^{i} y^{j} \mid i>0, j \in \mathbb{Z}\right\} \cup\{1\}$, a submonoid of the free abelian group $\operatorname{gr}(x, y)$ of rank two. It is easy to see that for a given $i>0$ and $j \in \mathbb{Z}$ the ideal $x^{i} y^{j} S$ contains the set $\left\{x^{k} y^{j} \mid k>i, j \in \mathbb{Z}\right\}$. Therefore $P=S \backslash\{1\}$ is nil modulo the ideal $x^{i} y^{j} S$. It follows that $S \backslash\{1\}$ is the only prime ideal of $S$. Moreover $\operatorname{rk}(S / P)=0<\operatorname{rk}(S)-1$, while $P$ is a minimal prime ideal of $S$. Clearly $K[P]$ is a prime ideal of $K[S]$. Let $P^{\prime}$ be the $K$-linear span of the set consisting of all elements of the form $x^{i}\left(y^{j}-y^{k}\right)$ with $i>0$ and $j, k \in \mathbb{Z}$. Then $P^{\prime}$ is an ideal of $K[S]$ and $K[S] / P^{\prime}$ is isomorphic to the polynomial algebra $K[x]$. Therefore $K[P]$ is a prime ideal of height two (note that $\operatorname{clKdim}(K[S])=\operatorname{rk}(S)=2)$.

The latter equalities also follow from the following result, which will be needed later in the paper. Let $T$ be a cancellative semigroup and $K$ a field. If $K[T]$ satisfies a polynomial identity then $\operatorname{clKdim}(K[T])=\operatorname{rk}(T)=\operatorname{GK}(K[T])$, [28, Theorem 23.4]. (By GK $(R)$ we denote the Gelfand-Kirillov dimension of a $K$-algebra $R$.)

On the other hand, for Noetherian semigroup algebras one can also give an example (see Example 3.3) showing that Theorem 1.1 cannot be extended to prime ideals of height exceeding one.

In order to give some applications to the behaviour of prime ideals of $S$ and those of $S \cap H$ (with $H$ a normal subgroup of finite index in $S S^{-1}$ ) we prove the following technical lemma.

Lemma 1.2 Let $S$ be a submonoid of a polycyclic-by-finite group, say with a group of quotients $G$. Assume that $S$ satisfies the ascending chain condition on right ideals. Then $G$ has a poly-(infinite cyclic) normal subgroup $H$ of finite index so that $S \cap H$ is $G$-invariant and $H / \mathrm{U}(S \cap H)$ is abelian. If $G$ is abelianby-finite then $H$ can be chosen to be an abelian subgroup.

Proof. It is well known that $G$ contains a characteristic subgroup $C$ that is poly-(infinite cyclic). Since $S$ satisfies the ascending chain condition on right ideals, $G$ contains a normal subgroup $F$ so that $G / F$ is abelian-by-finite and $F \subseteq S$. Hence $C \cap F$ is a normal subgroup of $G$ that is poly-(infinite cyclic), 
$C \cap F \subseteq \mathrm{U}(S)$ and $G /(C \cap F)$ is abelian-by-finite. It is thus sufficient to prove the result for the monoid $S / \sim_{C \cap F}$ and its group of quotients $G /(C \cap F)$. In other words we may assume that $G$ is abelian-by-finite.

So, let $A$ be a torsion free abelian and normal subgroup of finite index in $G$. Then $S \cap A$ is a finitely generated abelian monoid and its group of quotients is of finite index in $G$. Since $S$ satisfies the ascending chain condition on right ideals, we also know [22] that for every $g \in S$ and $s \in S$ there exists a positive integer $k$ so that $g s^{k} g^{-1} \in S$. Because $G=S \mathrm{Z}(S)^{-1}$ (again by the remark before Theorem [1.1), the latter property holds for all $g \in G$. As $S \cap A$ is finitely generated abelian and $G / A$ is finite, it follows that there exists a positive integer $n$ so that

$$
g(S \cap A)^{(n)} g^{-1} \subseteq S \cap A,
$$

for all $g \in G$, where by definition $(S \cap A)^{(n)}=\left\{s^{n} \mid s \in S \cap A\right\}$. Hence

$$
T=\bigcap_{g \in G} g(S \cap A)^{(n)} g^{-1}
$$

is a $G$-invariant submonoid of $S$. Because $A$ is the group of quotients of $S \cap A$, we get that each $g(S \cap A)^{(n)} g^{-1}$ has a group of quotients that is of finite index in $G$. Since there are only finitely many such conjugates, it is clear that $T T^{-1}$ is of finite index in $G$. Hence, the result follows.

The notion of height $\operatorname{ht}(P)$ of a prime ideal of a ring $R$ is well known. We now define the height $\operatorname{ht}(Q)$ of a prime ideal $Q$ of a monoid $S$. If $S$ does not have a zero element then $\operatorname{ht}(Q)$ is the maximal length $n$ of a chain $Q_{0} \subset Q_{1} \subset$ $Q_{2} \subset \cdots \subset Q_{n}=Q$, where $Q_{0}=\emptyset$ and $Q_{1}, \ldots, Q_{n-1}$ are prime ideals of $S$. On the other hand, if $S$ has a zero element, then $\operatorname{ht}(Q)$ is the maximal length of such a chain with all $Q_{i}$ prime ideals of $S, i \geq 0$. If such $n$ does not exist then we say that the height of $P$ is infinite.

Corollary 1.3 Let $S$ be a submonoid of a polycyclic-by-finite group, say with a group of quotients $G$, and let $K$ be a field. Assume that $S$ satisfies the ascending chain condition on right ideals. If $H$ is a torsion free normal subgroup of finite index in $G$, then

1. If $P$ is a prime ideal of $S$ then $P \cap H=Q_{1} \cap \cdots \cap Q_{n}$, where $Q_{1}, \ldots, Q_{n}$ are all the primes of $S \cap H$ that are minimal over $P \cap H$. Furthermore, $P=M \cap S$ for any prime ideal $M$ of $K[S]$ that is minimal over $K[P]$, $\operatorname{ht}(M)=\operatorname{ht}\left(K\left[Q_{1}\right]\right)=\cdots=\operatorname{ht}\left(K\left[Q_{n}\right]\right)$ and $P=\mathcal{B}\left(S\left(Q_{1} \cap \cdots \cap Q_{n}\right) S\right)$ (the prime radical of $\left.S\left(Q_{1} \cap \cdots \cap Q_{n}\right) S\right)$. If, furthermore, $H \cap S$ is $G$-invariant then $Q_{i}=Q_{1}^{g}$ for some $g \in G, 1 \leq i \leq n$.

2. If $S \cap H$ is $G$-invariant and $Q=Q_{1}$ is a prime ideal of $S \cap H$ then there exists a prime ideal $P$ of $S$ so that $P \cap H=Q_{1} \cap Q_{2} \cap \cdots \cap Q_{m}$, where $Q_{1}, \ldots, Q_{m}$ are all the prime ideals of $S \cap H$ that are minimal over $P \cap H$. One says that $P$ lies over $Q$. Moreover, each $Q_{i}=Q^{g_{i}}$ for some $g_{i} \in G$. 
3. Incomparability Suppose $S \cap H$ is $G$-invariant, $Q_{1}$ and $Q_{2}$ are prime ideals of $S \cap H$, and $P_{1}$ and $P_{2}$ are prime ideals of $S$. If $P_{1}$ lies over $Q_{1}$ and $P_{2}$ lies over $Q_{2}$ so that $Q_{1} \subseteq Q_{2}$ and $P_{1} \subseteq P_{2}$, then $P_{1}=P_{2}$ if and only if $Q_{1}=Q_{2}$.

4. Going up Assume $S \cap H$ is $G$-invariant. Suppose $Q_{2}$ is a prime ideal of $\mathrm{S} \cap \mathrm{H}$ and $\mathrm{P}_{2}$ is a prime ideal of $S$ lying over $Q_{2}$.

(a) If $Q_{1}$ is a prime ideal of $S \cap H$ containing $Q_{2}$ then there exists a prime ideal $P_{1}$ lying over $Q_{1}$ so that $P_{2} \subseteq P_{1}$.

(b) If $P_{1}$ is a prime ideal of $S$ containing $P_{2}$ then there exists a prime ideal $Q_{1}$ of $S \cap H$ containing $Q_{2}$ so that $P_{1}$ lies over $Q_{1}$.

5. Going down Assume $S \cap H$ is $G$-invariant. Suppose $Q_{1}$ is a prime ideal of $S \cap H$ and $P_{1}$ is a prime ideal of $S$ lying over $Q_{1}$.

(a) If $Q_{2}$ is a prime ideal of $S \cap H$ contained in $Q_{1}$ then there exists a prime ideal $P_{2}$ lying over $Q_{2}$ so that $P_{2} \subseteq P_{1}$.

(b) If $P_{2}$ is a prime ideal of $S$ contained in $P_{1}$ then there exists a prime ideal $Q_{2}$ of $S \cap H$ contained in $Q_{1}$ so that $P_{2}$ lies over $Q_{2}$.

Proof. The algebra $K[S]$ has a natural gradation by the finite group $G / H$. Its homogeneous component of degree $e$ (the identity of the group $G$ ) is the semigroup algebra $K[S \cap H]$. Let $P$ be a prime ideal of $S$. Let $M$ be a prime ideal of $K[S]$ minimal over $K[P]$. Note that then $K[S] / K[P]$ inherits a natural $G / H$ gradation, with component of degree $e$ the algebra $K[S \cap H] / K[P \cap H]$. Because of Theorem 17.9 in 32 on going-up and down on prime ideals of rings graded by finite groups, one gets that $K[S \cap H] \cap M=P_{1} \cap \cdots \cap P_{n}$, where $P_{1}, \ldots, P_{n}$ are all the prime ideals of $K[S \cap H]$ that are minimal over $K[P] \cap K[S \cap H]=K[P \cap H]$. Furthermore, $\operatorname{ht}\left(P_{i}\right)=\operatorname{ht}(M)$ for $1 \leq i \leq n$. Since $H$ is torsion free, we know that $K\left[P_{i} \cap H\right]$ is a prime ideal of $K[S \cap H]$ (see the introduction). Since it clearly contains $K[P \cap H]$, it follows that $P_{i}=K\left[Q_{i}\right]$, with $Q_{i}=P_{i} \cap H$. Furthermore, since $K[Q]$ is a prime ideal in $K[S \cap H]$ for every prime ideal $Q$ of $S \cap H$, it follows that $Q_{1}, \ldots, Q_{n}$ are all the prime ideals of $S \cap H$ minimal over $P \cap H$. So $K[S \cap H] \cap M=K\left[Q_{1} \cap \cdots \cap Q_{n}\right]$. Because $K[S \cap H] / K[P \cap H]$ is Noetherian, we know that its prime radical is nilpotent. Hence $\left(Q_{1} \cap \cdots \cap Q_{n}\right)^{k} \subseteq P \cap H$ for some positive integer $k$. Since $H$ is of finite index in $G$, it then also follows that $M \cap S$ is an ideal of $S$ that is nil modulo $S(P \cap H) S$. Since $K[S]$ is Noetherian, this yields that $(M \cap S)^{l} \subseteq S(P \cap H) S \subseteq P$, for some positive integer $l$. As $P$ is a prime ideal, we therefore obtain that $M \cap S=P, P \cap H=Q_{1} \cap \cdots \cap Q_{n}$ and $P=\mathcal{B}\left(S\left(Q_{1} \cap \cdots \cap Q_{n}\right) S\right)$.

Assume now that, furthermore, $S \cap H$ is $G$-invariant. For an ideal $I$ of $S \cap H$ put $I^{i n v}=\bigcap_{g \in G} I^{g}$, the largest invariant ideal of $S \cap H$ contained in $I$. Clearly $S I^{i n v}=I^{i n v} S$ is an ideal of $S$ and $S I^{i n v} \cap(S \cap H)=I^{i n v}$. It follows that

$$
S Q_{1}^{i n v} \cdots S Q_{n}^{i n v} \subseteq P .
$$


Hence $Q_{i}^{i n v} \subseteq P \cap(S \cap H)=Q_{1} \cap \cdots \cap Q_{n}$ for some $i$. Because $S \cap H$ is invariant, it follows that every $Q_{i}^{g}$ is a prime ideal of $S \cap H$ (of the same height as $Q_{i}$ ). Hence, for every $1 \leq j \leq n$ there exists $g \in G$ with $Q_{i}^{g}=Q_{j}$. This proves the first part of the result.

To prove the second part, let $Q$ be a prime ideal of $S \cap H$ and suppose that $S \cap H$ is $G$-invariant. Then, each $Q^{g}$ is a prime ideal of $S \cap H$ and ht $(Q)=$ ht $\left(Q^{g}\right)$. Now, if $Q^{\prime}$ is a prime ideal of $S \cap H$ containing $Q^{i n v}$, then $Q^{g} \subseteq Q^{\prime}$ for some $g \in G$. Hence, if $Q^{\prime}$ is a prime minimal over $Q^{i n v}$ then $Q^{g}=Q^{\prime}$. Clearly, for every $h \in G$, the prime $Q^{h}$ contains a prime ideal $Q^{\prime \prime}$ of $S \cap H$ minimal over $Q^{i n v}$. Hence, by the previous, $Q^{\prime \prime}=Q^{g} \subseteq Q^{h}$, for some $g$. Since $\operatorname{ht}\left(Q^{g}\right)=\operatorname{ht}\left(Q^{h}\right)$, it thus follows that $Q^{h}=Q^{\prime \prime}$. So, we have shown that the ideals $Q^{g}$ are precisely the prime ideals of $S \cap H$ that are minimal over $Q^{i n v}$. Since $H$ is torsion free we thus get (again see the introduction) that the ideals $K\left[Q^{g}\right]$ are precisely the prime ideals of $K[S \cap H]$ that are minimal over $K\left[Q^{i n v}\right]$. Since $S Q^{i n v}=Q^{i n v} S$ is an ideal of $S$, the algebra $K[S] / K\left[S Q^{i n v}\right]$ has a natural $G / H$-gradation, with $K[S \cap H] / K\left[Q^{i n v}\right]$ as component of degree $e$. Hence, by [32, Theorem 17.9], there exists a prime ideal $M$ of $K[S]$ that is minimal over $K\left[S Q^{i n v}\right]$ and $M \cap K[S \cap H]=K[Q] \cap P_{2} \cap \cdots \cap P_{m}$, where $K[Q], P_{2}, \ldots, P_{m}$ are all the prime ideals that are minimal over $M \cap K[S \cap H]$ (and these ideals are of the same height as $M$ ). Hence each $P_{i}$ is minimal over $K\left[Q^{i n v}\right]$ and thus is of the form $K\left[Q^{g}\right]$ for some $g$. It follows that $P=M \cap S$ is a prime ideal of $S$ so that $P \cap H$ is an intersection of $Q$ and some of the prime ideals $Q^{g}$, with $g \in G$. This proves part two.

Parts (3), (4) and (5) are now immediate consequences of parts (1) and (2) and of the corresponding results on going up and down for rings graded by finite groups (see for example [32, Theorem 17.9]).

A fundamental result (see [32, Theorem 19.6]) on the group algebra $K[G]$ of a polycyclic-by-finite group says that $\operatorname{clK} \operatorname{dim}(K[G])=\operatorname{pl}(G)$. For the definition on the plinth length $\operatorname{pl}(G)$ of $G$ we refer the reader also to 32. In the following result we determine relations between the considered invariants of $S$ and $K[S]$ for Noetherian semigroup algebras $K[S]$ of submonoids $S$ of polycyclic-by-finite groups.

Corollary 1.4 Let $S$ be a submonoid of a polycyclic-by-finite group and let $K$ be a field. Assume that $S$ satisfies the ascending chain condition on right ideals and let $G$ be its group of quotients. Let $F$ be a normal subgroup of $G$ so that $F \subseteq S, \mathrm{U}(S) / F$ is finite and $G / F$ is abelian-by-finite. The following properties hold.

1. $\operatorname{dim}(S)=\operatorname{rk}(G / F)$.

2. $\operatorname{dim}(S)=\operatorname{dim}(S \cap W)$ for any normal subgroup $W$ of $G$ of finite index.

3. $\operatorname{Spec}(S)$ is finite.

4. $\operatorname{clKdim}(K[S])=\operatorname{dim}(S)+\operatorname{pl}(\mathrm{U}(S))$. 
Proof. Because of Lemma 1.2, the group $G$ has a poly-(infinite cyclic) normal subgroup $H$ of finite index so that $S \cap H$ is $G$-invariant and $H / F$ is abelian for some normal subgroup $F$ of $H$ that is contained in $S$. Parts (1-5) of Corollary 1.3 easily yield that $\operatorname{dim}(S)=\operatorname{dim}(S \cap H)$. It also easily is verified that $\operatorname{dim}(S \cap H)=$ $\operatorname{dim}(T)$ where $T=(S \cap H) / \sim_{F}$. Clearly $T T^{-1}$ is abelian and $T T^{-1}=H / F$ because $[G: H]<\infty$. Since $\mathrm{U}(S) / F$ is finite, we obtain that $\mathrm{U}(T)$ is finite. Hence $\operatorname{dim}(T)=\operatorname{rk}(T)$ (see 22]). So part (1) follows.

To prove part (2) let $W$ be a normal subgroup of finite index in $G$. Then, $S \cap W$ inherits the assumptions on $S$ and $W$ is the group of quotients of $S \cap W$. So, from part (1) we obtain that $\operatorname{dim}(S)=\operatorname{rk}(G / F)=\operatorname{rk}(W /(F \cap W))=$ $\operatorname{dim}(S \cap W)$ and thus $\operatorname{dim}(S)=\operatorname{dim}(S \cap W)$.

It easily is seen that there is a natural bijective map between $\operatorname{Spec}(S)$ and $\operatorname{Spec}\left(S / \sim_{F}\right)$. Hence to prove part (3) we may assume that $S S^{-1}$ is abelianby-finite. Because of Lemma 1.2 and part (1) of Corollary [1.3 we obtain that $\operatorname{Spec}(S)$ is finite if the corresponding property holds for finitely generated abelian monoids $A=\left\langle a_{1}, \ldots, a_{n}\right\rangle$. This is obviously satisfied. Indeed, if $P \in \operatorname{Spec}(A)$ then $A \backslash P$ is a submonoid of $A$ and $a_{i} \in P$ for some $i$. It follows that $A \backslash P$ is generated by a proper subset of $\left\{a_{1}, \ldots, a_{n}\right\}$, whence $|\operatorname{Spec}(A)|<\infty$.

Finally, we prove part (4). From [22, Corollary 4.4] we know that

$$
\operatorname{clKdim}(K[S])=\operatorname{rk}(G / F)+\operatorname{pl}(\mathrm{U}(S)) .
$$

So the statement follows at once from part (1).

In the following proposition it is shown that the prime spectra of $S$ and $S / \sim_{\Delta^{+}\left(S S^{-1}\right)}$ can be identified.

Proposition 1.5 Let $S$ be a submonoid of a polycyclic-by-finite group $G=$ $S S^{-1}$ and assume that $S$ satisfies the ascending chain condition on right ideals. Let $H=\Delta^{+}(G)$, the maximal finite normal subgroup of $G$. Let $\sim$ be the congruence relation on $S$ determined by $H$, that is, $s \sim t$ if and only if $s H=t H$. Then the map $\operatorname{Spec}(S) \longrightarrow \operatorname{Spec}(S / \sim)$, defined by $P \mapsto P / \sim$, is a bijection.

Proof. Let $\varphi: S \longrightarrow S / \sim \subseteq G / H$ be the natural epimorphism. Let $P$ be a prime ideal of $S$.

We claim that $P=\varphi^{-1}(\varphi(P))$. One inclusion is obvious. To prove the converse, let $x \in \varphi^{-1}(\varphi(P))$. Then there exists $p \in P$ such that $x \sim p$. So $x=p h$ for some $h \in H$. Let $s \in S$. Then

$$
(h s p)^{n}=h h^{s p} h^{(s p)^{2}} \cdots h^{(s p)^{n-1}}(s p)^{n},
$$

for every $n \geq 1$. Because $h \in H$, there exists a positive integer $k$ so that $h^{(s p)^{k}}=h$. Define $n=(k-1)|H|+1$. Then

$$
(h s p)^{n}=\left(h h^{s p} h^{(s p)^{2}} \cdots h^{(s p)^{k-1}}\right)^{|H|}(s p)^{n}=(s p)^{n} \in P .
$$


It follows that $x S$ is nil modulo $P$. Because $K[S]$ is Noetherian, from [17, Theorem 5.18] we get that $x \in P$. This proves the claim.

The claim easily implies that $\varphi(P)$ is a prime ideal of $S$ and the statement follows.

We can now prove for the semigroups under consideration an analogue of Schelter's theorem on prime affine algebras that satisfy a polynomial identity; this on its turn yields the catenary property (see for example [27, Theorem 13.10.12 and Corollary 13.10.13]).

Proposition 1.6 Let $S$ be a submonoid of a polycyclic-by-finite group, say with a group of quotients $G$. Assume that $S$ satisfies the ascending chain condition on right ideals. Let $P$ be a prime ideal of $S$. Then $\operatorname{dim}(S / P)+\operatorname{ht}(P)=\operatorname{dim}(S)$. Furthermore, if $\mathrm{U}(S)$ is finite, then $\operatorname{dim}(S / P)=\operatorname{rk}(S / P)$.

Proof. Again let $F$ be a normal subgroup of $G$ so that $F \subseteq \mathrm{U}(S),[\mathrm{U}(S)$ : $F]<\infty, S \cap F$ is finitely generated and $G / F$ is abelian-by-finite. Because of the natural bijection between $\operatorname{Spec}(S)$ and $\operatorname{Spec}\left(S / \sim_{F}\right)$, we may replace $S$ by $S / \sim_{F}$ and thus we may assume that $G$ is abelian-by-finite and $\mathrm{U}(S)$ is finite. Hence, by Lemma 1.2 $G$ contains a normal torsion free abelian subgroup $A$ so that $[G: A]<\infty, T=S \cap A$ is finitely generated and $G$-invariant.

We now first prove that $\operatorname{dim}(T / Q)+\operatorname{ht}(Q)=\operatorname{dim}(T)$ for a prime ideal $Q$ in the abelian monoid $T$. Since $A$ is torsion free, we know (see the introduction) that $K[Q]$ is a prime ideal of $K[T]$ (which is of height one if $Q$ is a minimal prime of $T$ ). Hence (by Schelter's result for finitely generated commutative algebras) $\operatorname{clKdim}(K[T])=\operatorname{clKdim}(K[T] / K[Q])+\operatorname{ht}(K[Q])$. Note that $T \backslash Q$ is a submonoid of $A$ and $\operatorname{clKdim}(K[T] / K[Q])=\operatorname{clKdim}(K[T \backslash Q]$ ) (we will several times use this fact without specific reference). Consequently, by Corollary 1.4 $\operatorname{dim}(T)=\operatorname{dim}(T \backslash Q)+\operatorname{ht}(K[Q])$. Since $\operatorname{dim}(T \backslash Q)=\operatorname{dim}(T / Q)$, we need to prove that $\operatorname{ht}(K[Q])=\operatorname{ht}(Q)$. We prove this by induction on $\operatorname{ht}(Q)$ (note that by Corollary 1.4 the prime spectrum of $T$ is finite and hence every prime contains a minimal prime ideal of $T$ ). If $\operatorname{ht}(Q)=1$ then the statement holds. So, assume $\operatorname{ht}(Q)>1$. Let $Q_{1}$ be a prime of height one contained in $Q$. Then, by the induction hypothesis, $\operatorname{ht}\left(Q / Q_{1}\right)=\operatorname{ht}\left(K\left[Q / Q_{1}\right]\right)$. Since $K\left[\left(T / Q_{1}\right) /\left(Q / Q_{1}\right)\right] \cong K[T / Q]$, we thus get from Schelter's result that $\operatorname{clKdim}(K[T])-1=\operatorname{clKdim}\left(K\left[T / Q_{1}\right]\right)=\operatorname{clKdim}(K[T / Q])+\operatorname{ht}\left(Q / Q_{1}\right)=$ $\operatorname{clKdim}(K[T])-\operatorname{ht}(K[Q])+\mathrm{ht}\left(Q / Q_{1}\right)$. Hence ht $(K[Q])=\operatorname{ht}\left(Q / Q_{1}\right)+1 \leq \operatorname{ht}(Q)$. Since $K[M]$ is prime in $K[T]$ if $M$ is prime in $T$, it is clear that $\operatorname{ht}(Q) \leq$ $\operatorname{ht}(K[Q])$. Hence we obtain that ht $(K[Q])=\mathrm{ht}(Q)$, as desired.

Now let $P$ be a prime ideal of $S$. Because of Corollary 1.3. $\operatorname{dim}(S)=$ $\operatorname{dim}(S \cap A), \operatorname{dim}(S / P)=\operatorname{dim}(T / Q)$ and $\operatorname{ht}(P)=\operatorname{ht}(Q)$, where $Q$ is a prime ideal of $T$ and $P$ lies over $Q$. From the previous it thus follows that

$$
\operatorname{dim}(S / P)+\operatorname{ht}(P)=\operatorname{dim}(S) .
$$

So, only the last part of the statement of the result remains to be proven. Let $Q_{1}=Q$ and write $P \cap T=Q_{1} \cap \cdots \cap Q_{n}$, where $Q_{1}, \ldots, Q_{n}$ are all primes minimal 
over $P \cap T$. We know that $\operatorname{clK} \operatorname{dim}\left(K\left[T / Q_{i}\right]\right)=\operatorname{rk}\left(T / Q_{i}\right)$. Furthermore, because $T T^{-1}$ is torsion free and $K[T]$ is Noetherian, we also know that $K\left[Q_{i}\right]$ is a prime ideal of $K[T]$ that is minimal over $K[P \cap T]$ (see the introduction). Hence, $K\left[Q_{i} /(P \cap T)\right]$ is a minimal prime ideal in the finitely generated commutative algebra $K[T /(P \cap T)]$. It follows that

$$
\begin{aligned}
\operatorname{clKdim}(K[T /(P \cap T)]) & =\operatorname{clKdim}\left(K\left[T / Q_{i}\right]\right)+\operatorname{ht}\left(K\left[Q_{i} /(P \cap T)\right]\right) \\
& =\operatorname{clKdim}\left(K\left[T / Q_{i}\right]\right)=\operatorname{rk}\left(T / Q_{i}\right) .
\end{aligned}
$$

Since $K[T /(P \cap T)]$ is a finitely generated commutative algebra, it is well known that $\operatorname{clKdim}(K[T /(P \cap T)])=\operatorname{GK}(K[T /(P \cap T)])$ (see for example [26, Theorem 4.5]). From [28, Theorem 23.14]) it follows that

$$
\operatorname{rk}(T /(P \cap T))=\operatorname{clKdim}(K[T /(P \cap T)]) .
$$

Using (1) we thus get

$$
\begin{aligned}
\operatorname{rk}(T /(P \cap T)) & =\operatorname{clKdim}\left(K[T] /\left(K\left[Q_{1}\right] \cap \cdots \cap K\left[Q_{n}\right]\right)\right) \\
& =\sup \left\{\operatorname{clKdim}\left(K\left[T / Q_{i}\right]\right) \mid 1 \leq i \leq n\right\} \\
& =\operatorname{clKdim}(K[T / Q])=\operatorname{rk}(T / Q) .
\end{aligned}
$$

Since $T T^{-1}$ is of finite index in $S S^{-1}$, it follows that

$$
\operatorname{rk}(S / P)=\operatorname{rk}(T /(T \cap P))=\operatorname{rk}(T / Q) .
$$

Because $\mathrm{U}(T / Q)$ is finite, Corollary 1.4 yields that $\operatorname{rk}(T / Q)=\operatorname{dim}(T / Q)$ and thus we get that

$$
\begin{aligned}
\operatorname{rk}(S / P)=\operatorname{rk}(T / Q) & =\operatorname{dim}(T / Q)=\operatorname{dim}(T)-\operatorname{ht}(Q) \\
& =\operatorname{dim}(S)-\operatorname{ht}(P)=\operatorname{dim}(S / P) .
\end{aligned}
$$

This finishes the proof.

\section{Maximal Orders}

In this section we describe when a semigroup algebra $K[S]$ of a cancellative submonoid $S$ of a polycyclic-by-finite group $G$ is a prime Noetherian maximal order that satisfies a polynomial identity. In case $G$ is torsion free such a result was obtained in 21] and in case $S=G$ this was done by Brown in [3, 4, (even without the restriction that $K[S]$ has to be PI).

For completeness' sake we recall some notation and terminology on (maximal) orders. We state these in the semigroup context (see for example [16] and 34 ) as these are basically the same as in the more familiar ring case. A cancellative monoid $S$ which has a left and right group of quotients $G$ is called an order. Such a monoid $S$ is called a maximal order if there does not exist 
a submonoid $S^{\prime}$ of $G$ properly containing $S$ and such that $a S^{\prime} b \subseteq S$ for some $a, b \in G$. For subsets $A, B$ of $G$ we define $\left(A:_{l} B\right)=\{g \in G \mid g B \subseteq A\}$ and by $\left(A:_{r} B\right)=\{g \in G \mid B g \subseteq A\}$. Note that $S$ is a maximal order if and only if $\left(I:_{l} I\right)=\left(I:_{r} I\right)=S$ for every fractional ideal $I$ of $S$. The latter means that $S I S \subseteq I$ and $c I, I d \subseteq S$ for some $c, d \in S$. If $S$ is a maximal order, then $\left(S:_{l} I\right)=\left(S:_{r} I\right)$ for any fractional ideal $I$; we simply denote this fractional ideal by $(S: I)$ or by $I^{-1}$. Recall that then $I$ is said to be divisorial if $I=I^{*}$, where $I^{*}=(S:(S: I))$. The divisorial product $I * J$ of two divisorial ideals $I$ and $J$ is defined as $(I J)^{*}$. Also recall that a fractional ideal is said to be invertible if $I J=J I=S$ for some fractional ideal $J$ of $S$. In this case $J=I^{-1}$ and $I$ is a divisorial ideal.

Recall then that (see for example [34]) a cancellative monoid $S$ is said to be a Krull order if and only if $S$ is a maximal order satisfying the ascending chain condition on divisorial integral ideals (the latter are the fractional ideals contained in $S$ ). In this case the set $D(S)$ of divisorial fractional ideals is a free abelian group for the * operation. If $S S^{-1}$ is abelian-by-finite then (as said before, see [25, Lemma 1.1]) every ideal of $S$ contains a central element and it follows that the minimal primes of $S$ form a free basis for $D(S)$, 34.

Similarly a prime Goldie ring $R$ is said to be a Krull order if $R$ is a maximal order that satisfies the ascending chain condition on divisorial integral ideals. Although there are several notions of noncommutative Krull orders, for rings satisfying a polynomial identity all these notions are the same.

In the next theorem we collect some of the essential properties of these orders. For details we refer the reader to [5, 6]. For a ring $R$ and an Ore set $C$ of regular elements in $R$ we denote by $R_{C}$ the classical localization of $R$ with respect to $C$. The classical ring of quotients of a prime Goldie ring $R$ is denoted by $Q_{c l}(R)$. The prime spectrum of $R$ is denoted by $\operatorname{Spec}(R)$, the set of height one prime ideals of $R$ by $X^{1}(R)$.

Theorem 2.1 Let $R$ be a prime Krull order satisfying a polynomial identity. Then the following properties hold.

1. The divisorial ideals form a free abelian group with basis $X^{1}(R)$, the height one primes of $R$.

2. If $P \in X^{1}(R)$ then $P \cap \mathrm{Z}(R) \in X^{1}(\mathrm{Z}(R))$, and furthermore, for any ideal $I$ of $R, I \subseteq P$ if and only if $I \cap \mathrm{Z}(R) \subseteq P \cap \mathrm{Z}(R)$.

3. $R=\bigcap R_{Z(R) \backslash P}$, where the intersection is taken over all height one primes of $R$, and every regular element $r \in R$ is invertible in almost all (that is, except possibly finitely many) localizations $R_{\mathrm{Z}(R) \backslash P}$. Furthermore, each $R_{\mathrm{Z}(R) \backslash P}$ is a left and right principal ideal ring with a unique nonzero prime ideal.

4. For a multiplicatively closed set of ideals $\mathcal{M}$ of $R$, the (localized) ring $R_{\mathcal{M}}=\left\{q \in Q_{c l}(R) \mid I q \subseteq R\right.$, for some $\left.I \in \mathcal{M}\right\}$ is a Krull order, and

$$
R_{\mathcal{M}}=\bigcap R_{\mathrm{Z}(R) \backslash P},
$$


where the intersection is taken over those height one primes $P$ for which $R_{\mathcal{M}} \subseteq R_{\mathrm{Z}(R) \backslash P}$.

Next we prove some necessary condition for $K[S]$ to be a prime Noetherian maximal order that satisfies a polynomial identity.

Lemma 2.2 Let $S$ be a submonoid of an abelian-by-finite group $G=S S^{-1}$ and let $K$ be a field. Let $A$ be an abelian subgroup that is normal and of finite index in $G$ and let $P$ be a minimal prime ideal of $S$. The following properties hold.

1. If $S$ is a maximal order then $S \cap A$ is $G$-invariant and $S \cap A$ is a maximal order in its group of quotients.

2. If $K[S]$ is a prime Noetherian maximal order, then $S$ is a maximal order, $S \cap A$ and $P \cap A$ are $G$-invariant.

Proof. By [21, Lemma 2.1], if $S$ is a maximal order then $S \cap A$ is $G$-invariant and it is a maximal order in its group of quotients. Assume now that $K[S]$ is a prime Noetherian maximal order. It is straightforward (as in the proof of Lemma 3.3 in [21]) to verify that then $S$ is a maximal order, and thus $S \cap A$ is $G$-invariant. Let $P$ be a minimal prime ideal of $S$. Of course, $A \subseteq \Delta(G)$ and thus $P \cap A=(P \cap \Delta(G)) \cap(A \cap S)$. Hence to prove that $P \cap A$ is $G$ invariant, we may assume in the remainder that $A=\Delta(G)$. Indeed, since $K[S]$ is prime, by [28, Theorem 7.19], $K[G]$ is prime. Then, from [31, Theorem 4.2.10] it follows that $\Delta(G)$ is abelian. Now, by Corollary 1.3, $P \cap A=Q_{1} \cap \cdots \cap Q_{n}$, an intersection of minimal primes of $A$ that are $G$-conjugate. We need to show that $\left\{Q_{1}, \ldots, Q_{n}\right\}=\left\{Q_{1}^{g} \mid g \in G\right\}$. Suppose the contrary, so assume $Q^{\prime}$ is a minimal prime of $S \cap A$ that is conjugate to $Q_{1}$ but is different from all $Q_{i}$, for $1 \leq i \leq n$. Then by Corollary 1.3, there exists a prime ideal $P^{\prime}$ of $S$ so that $P^{\prime} \cap A=Q^{\prime} \cap Q_{2}^{\prime} \cap \cdots \cap Q_{m}^{\prime}$ for some minimal primes $Q_{2}^{\prime}, \ldots, Q_{m}^{\prime}$ of $S \cap A$. Because of Theorem 1.1, both $K[P]$ and $K\left[P^{\prime}\right]$ are distinct height one prime ideals of $K[S]$. As, by assumption, $K[S]$ is a prime PI Noetherian maximal order, it follows from Theorem 2.1 that there exists a central element of $K[S]$ that belongs to $K\left[P^{\prime}\right]$ but not to $K[P]$. Since central elements of $K[S]$ are linear combinations of finite conjugacy class sums of $K[G]$ we get that $P^{\prime}$ contains a $G$-conjugacy class $C$ that does not belong to $P$. Since $A=\Delta(G)$, we thus get that $C \subseteq A$. Hence, $C \subseteq Q^{\prime}$. As $Q^{\prime}$ is a $G$-conjugate of $Q_{1}$ it follows that $C \subseteq Q_{1} \cap \cdots \cap Q_{n}=P \cap A$, a contradiction.

We need one more lemma in order to prove the main theorem of this section. If $\alpha=\sum_{s \in S} k_{s} s$ (with each $k_{s} \in K$ ) then we denote by $\operatorname{supp}(\alpha)=\{s \in S \mid$ $\left.k_{s} \neq 0\right\}$ the support of $\alpha$.

Lemma 2.3 Let $S$ be a submonoid of an abelian-by-finite group $G=S S^{-1}$. Let $K$ be a field and suppose $K[S]$ is Noetherian. Let $A$ be a normal abelian subgroup of finite index in $G$. Assume that $P$ is a prime ideal of $S$ so that $K[P]$ is a prime ideal of $K[S]$ and $P \cap A$ is G-invariant. Also assume that $S \cap A$ is $G$-invariant. If $J$ is an ideal of $S$ not contained in $P$ then $J \cap A$ contains a $G$-conjugacy class $D$ such that $D \nsubseteq P$ (and thus clearly $D \subseteq(J \cap A) \backslash P$ ). 
Proof. We may assume that $J$ is a proper ideal of $S$. The prime algebra $R=K[S] / K[P]$ has a natural $G / A$-gradation, with identity component $R_{e}=$ $K[S \cap A] / K[P \cap A]$, a semiprime commutative algebra. Let $L$ be a nonzero ideal of $R$. It then follows from [10, Theorem 1.7] that $r_{e} \in L \cap R_{e}$, for some regular element $r_{e}$ of $R_{e}$. Because of the assumptions, $G$ acts by conjugation in $R_{e}$. Clearly, $r$ has only finitely many such conjugates, say $r_{1}, \ldots, r_{m}$ and $r_{1} \cdots r_{m} \in L \cap R_{e}$ is central and nonzero. So, $L$ contains a non-trivial element in $R_{e} \cap \mathrm{Z}(R)$.

We apply the above to the ideal $L=(K[J]+K[P]) / K[P]$. So, let $\alpha \in$ $K[J \cap A]$ be such that the image $\bar{\alpha} \in K[S] / K[P]$ is nonzero and lies in the center. So $\alpha$ is regular modulo $K[P]$. We may assume that $\operatorname{supp}(\alpha) \cap P=\emptyset$. Note also that $1 \notin \operatorname{supp}(\alpha)$. Write $\alpha=\alpha_{1}+\cdots+\alpha_{q}$, where $\alpha_{i}$ have supports contained in different $G$-conjugacy classes. Then $g \alpha_{1}+\cdots+g \alpha_{q}-\left(\alpha_{1} g+\cdots+\alpha_{q} g\right) \in K[P]$ for every $g \in S$. Clearly, $g \alpha_{i}$ and $\alpha_{j} g$ have disjoint supports if $i \neq j$. So we must have $g \alpha_{i}-\alpha_{i} g \in K[P]$ for every $i$. Then every $\alpha_{i}$ also lies in the center modulo $K[P]$ and $\operatorname{supp}\left(\alpha_{i}\right)$ is contained in a $G$-conjugacy class $D_{i}$. By the hypothesis, $D_{i} \subseteq(S \cap A) \backslash P$. Hence, replacing $\alpha$ by $\alpha_{1}$, we may assume that $\alpha=\alpha_{1}$. Write $D=D_{1}$.

Because of the assumptions and Corollary 1.3, $P \cap A=Q_{1} \cap \cdots \cap Q_{n}$ where $Q_{1}, \ldots, Q_{n}$ is a full orbit of conjugate primes in $S \cap A$. For $1 \leq i \leq n$ let $A_{i}=\left(\bigcap_{j, j \neq i} Q_{j}\right) \backslash Q_{i}$. Each $A_{i}$ is a subsemigroup of $A \backslash P$. Each $g \in G$ permutes the sets $A_{1}, \ldots, A_{n}$ (by conjugation). Let $I=\bigcup_{i=1}^{n} A_{i} \cup(P \cap A)$. Then $I$ is $G$-invariant, $S I=I S$ and $S I \cap A=I$. Replacing $J$ by $J \cap S I$ we may assume that $J \subseteq S I$ and thus also $\alpha \in K[J \cap A] \subseteq K[S I \cap A]=K[I]$. Hence, we can write $\alpha=\gamma_{1}+\cdots+\gamma_{n}$, with $\operatorname{supp}\left(\gamma_{i}\right) \subseteq A_{i}$ for $i=1, \ldots, n$. Notice that $\operatorname{supp}\left(\gamma_{i}\right) \neq \emptyset$ for every $i$. Indeed, suppose the contrary, that is, assume $\gamma_{i}=0$ for some $i$. Then, $\alpha A_{i} \subseteq P$, in contradiction with the regularity of $\alpha$ modulo $K[P]$. So, indeed, $\operatorname{supp}\left(\gamma_{i}\right) \neq \emptyset$ for every $i$. Let $E_{i}=D \cap A_{i}$. Since also $\operatorname{supp}\left(\gamma_{i}\right) \subseteq D$, we get that $\emptyset \neq \operatorname{supp}\left(\gamma_{i}\right) \subseteq E_{i}$. Put $a_{i}=\prod_{x \in E_{i}} x$. Clearly $a_{i} \in A_{i}$. It follows that $a_{1}+\cdots+a_{n} \in K[J]$. Let $1 \leq i \leq n$ and $g \in G$. Then $g^{-1} A_{i} g=A_{j}$ for some $j$. Hence $g^{-1} E_{i} g=E_{j}$ and thus $g^{-1} a_{i} g=a_{j}$. So, conjugation by elements of $G$ permutes $a_{1}, \ldots, a_{n}$. Therefore the result follows.

Recall from [3, 32 that a group $G$ is dihedral free if, for every subgroup $D$ of $G$ isomorphic to the infinite dihedral group, the normalizer $N_{G}(D)$ of $D$ in $G$ has infinite index.

Theorem 2.4 Let $K$ be a field and let $S$ be a submonoid of a finitely generated abelian-by-finite group. Let $A$ be an abelian normal subgroup of finite index in $G=S S^{-1}$. The following conditions are equivalent.

1. $K[S]$ is a prime Noetherian maximal order.

2. $S$ is a maximal order that satisfies the ascending chain condition on right ideals, $\Delta^{+}(G)=\{1\}, G$ is dihedral free and for every minimal prime ideal $P$ of $S$ the set $A \cap P$ is $G$-invariant. 
Proof. Assume that $K[S]$ is a prime Noetherian maximal order. Then, by Theorem [2.1 the localization $K[G]=K\left[S \mathrm{Z}(S)^{-1}\right]$ of $K[S]$ also is a maximal order. Because of Brown's result on the description of group algebras of polycyclic-by-finite groups that are maximal orders, the latter holds if and only if $\Delta^{+}(G)=\{1\}$ and $G$ is dihedral free. Lemma 2.2 then yields that the other conditions listed in (2) hold as well.

Conversely, assume that condition (2) holds. The assumption on $\Delta^{+}(G)$ yields that $K[G]$ and thus $K[S]$ is prime. Because $S$ is a maximal order, Lemma 2.2 gives that $S \cap A$ is $G$-invariant for any abelian normal subgroup $A$ of $G$ of finite index. To prove that $K[S]$ is a maximal order one can follow the lines of the proof of Theorem 3.5 in [21]. We now give a simplified proof.

We begin by showing that if $P$ is a minimal prime ideal of $S$ then the localized ring $R=K[S]((\mathrm{Z}(K[S]) \cap K[A]) \backslash K[P])^{-1}$ is a maximal order. To do so, we show that $R$ is a local ring with unique maximal ideal $R P$ and so that $R P$ is invertible and every proper nonzero ideal of $R$ is of the form $(R P)^{n}$ for some positive integer $n$. First we show that $R P$ is the only height one prime ideal of $R$. Of course if $Q^{\prime}$ is a height one prime ideal of $R$ then $Q=K[S] \cap Q^{\prime}$ is a height one prime ideal of $K[S]$ that does not intersect $(\mathrm{Z}(K[S]) \cap K[A]) \backslash K[P]$. Because of Theorem 1.1] either $Q=K[S \cap Q]$ or $S \cap Q=\emptyset$. Because of Lemma 2.3, the former implies that $S \cap Q \subseteq P$ and thus $Q=K[P]$, as desired. So assume $S \cap Q=$ $\emptyset$, or equivalently, $A \cap Q=\emptyset$. Because $S \cap A$ is $G$-invariant and $Q$ does not contain homogeneous elements, it follows (see for example Lemma 7.1.4 in 24]) that $\bar{Q}=\bigcap_{g \in G} g^{-1}(Q \cap K[A]) g$ is not contained in $K[P \cap A]$. As $\bar{Q}$ is $G$-invariant, we get that $K[S] \bar{Q}$ is an ideal of $K[S]$. So $(K[S] \bar{Q}+K[P]) / K[P]$ is a nonzero ideal of the Noetherian algebra $K[S] / K[P]$. This algebra has a natural $G / A$ gradation, with component of degree $e$ the semiprime algebra $K[S \cap A] / K[P \cap A]$. By Theorem 1.7 in [10, the ring $K[S] / K[P]$ has a classical ring of quotients that is obtained by inverting the regular elements of $K[S \cap A] / K[P \cap A]$. Hence the ideal $(K[S] \bar{Q}+K[P]) / K[P]$ contains a regular element $\bar{\alpha}$ that is contained in $K[S \cap A] / K[P \cap A]$. Since $K[S \cap A]$ and $K[P \cap A]$ are $G$-invariant, conjugation induces an action of $G$ on $K[S \cap A] / K[P \cap A]$. Hence the product of the finitely many conjugates of $\bar{\alpha}$ also belongs to $K[S \cap A] / K[P \cap A]$. Since this element is central, we thus may assume that $\bar{\alpha}$ also is central in $K[S] / K[P]$ and clearly $\bar{\alpha} \in(\bar{Q}+K[P \cap A]) / K[P \cap A]$. Write $\bar{\alpha}=\gamma+K[P \cap A]$ for some $\gamma \in \bar{Q}$. Let $\beta$ be the product of the distinct conjugates of $\gamma$. Then $\beta+K[P \cap A]=\gamma^{m}+K[P \cap A]$ for some positive integer $m$. Since $K[P \cap A]$ is a semiprime ideal in $K[S \cap A]$, it follows that $\beta \notin K[P \cap A]$. Hence $\beta \in(Q \cap \mathrm{Z}(K[S])) \backslash K[P]$, a contradiction. This implies that indeed $R P$ is the only height one prime ideal of $R$.

Because of Lemma 2.2. $S \cap A$ is a finitely generated maximal order. Hence we know that $K[S \cap A]$ is a Noetherian maximal order (1, 2]) and thus it is well known (or use Theorem 2.1) that $K[S \cap A]\left((\mathrm{Z}(K[S] \cap K[A]) \backslash P)^{-1}\right.$ is a Noetherian maximal order with only finitely many height one prime ideals. Hence it is a principal ideal domain (see for example [13]) and thus it has prime dimension one. As this is the component of degree $e$ of the $G / A$-graded ring $R$, from [32, Theorem 17.9] it follows that $R$ also has dimension one. Hence $R P$ is 
the only maximal ideal of $R$. As $R$ also is a PI algebra, we then obtain that $R P$ is the Jacobson radical of $R$ and thus, by [17, Theorem 8.12], $\bigcap_{n}(R P)^{n}=\{0\}$. Also note that $P(S: P)$ is an ideal of $S$ that is not contained in $P$. Hence $R P(S: P)=R$ and thus the unique maximal ideal $R P$ is an invertible ideal of $R$. It then easily follows that every proper nonzero ideal of $R$ is of the form $(R P)^{n}$ for some unique positive integer $n$. This proves the desired properties of $R$.

Let $\alpha=\sum_{i=1}^{n} k_{i} g_{i} \in K[G]$, where $0 \neq k_{i} \in K$ and $g_{i} \in G$ for each $1 \leq i \leq n$ and $g_{i} \neq g_{j}$ for $i \neq j$ We now show that if $\alpha \in K[G] \cap \bigcap_{P} K[S]((\mathrm{Z}(K[S]) \cap$ $K[A]) \backslash K[P])^{-1}$ (where the intersection runs over all minimal primes $P$ of $S$ ) then $\alpha \in K[S]$. We prove this by induction on $n$. If $n=1$ then $\alpha=k_{1} g_{1}$. For each minimal prime $P$ of $S$ there then exists a central element $\delta$ of $K[S]$ that belongs to $K[A] \backslash P$ so that $\delta k_{1} g_{1} \in K[S]$. Hence there is a $G$-conjugacy class $C(P)$ so that $C \subseteq(S \cap A) \backslash P$ and $C(P) g_{1} \subseteq S$. Let $C=\bigcup_{P} C(P)$. Then $S C g_{1} \subseteq S$ and $S C$ is an ideal of $S$ that is not contained in any of the minimal prime ideals of $S$. Since $S$ is a maximal order, it follows that $g_{1} \in S$, as desired. Now assume $n>1$. Since $\bigcap_{P} K[S]((\mathrm{Z}(K[S]) \cap K[A]) \backslash K[P])^{-1}$ is a $G / A$-graded ring, the induction hypothesis yields that we may assume that $\alpha$ is $G / A$-homogeneous, that is, each $g_{i} g_{j}^{-1} \in A$. Since this statement holds for any normal abelian subgroup of $G$ of finite index and because $A$ is residually finite, we get that $g_{i}=g_{j}$ for all $i=j$. Hence we may assume $n=1$ and thus by the above $\alpha \in K[S]$.

So we have shown that $K[S]=K[G] \cap \bigcap_{P} K[S]((\mathrm{Z}(K[S]) \cap K[A]) \backslash K[P])^{-1}$. Recall that by Theorem $\mathrm{F}$ in $[3$, since $G$ is dihedral-free, $K[G]$ is a maximal order. Since also each $K[S]((\mathrm{Z}(K[S]) \cap K[A]) \backslash K[P])^{-1}$ is a maximal order and a central localization of $K[S]$, it follows that $K[S]$ is a maximal order. This finishes the proof.

\section{Constructing examples}

Theorem 2.4 reduces the problem of determining when $K[S]$ is a prime Noetherian maximal order to the algebraic structure of $S$. It hence provides a strong tool for constructing new classes of such algebras. For some examples the required conditions on $S$ can easily be verified, but on the other hand, for some examples this still requires substantial work. In this section this is illustrated with some concrete constructions.

A first class of examples consists of algebras defined by monoids of $I$-type (see [14, 24). Recall that, in particular, these are quadratic algebras $R$ with a presentation defined by $n$ generators $x_{1}, \ldots, x_{n}$ and with $\left(\begin{array}{l}n \\ 2\end{array}\right)$ relations of the form $x_{i} x_{j}=x_{k} x_{l}$ so that every word $x_{i} x_{j}$ appears at most once in one of the defining relations. Clearly $R=K[S]$, where $S$ is the monoid defined by the same presentation. It turns out that $S$ has a group of quotients $G$ that is torsion free and has a free abelian subgroup $A$ of finite index. Furthermore, for any minimal prime ideal $P$ of $S$ one has that $P=S s=s S, P \cap A=(S \cap A) a$, 
for some $s \in S, a \in A$, and $P \cap A$ is $G$-invariant. Using Theorem 2.4 we then immediately recover the known result that $R$ is a maximal order. The only noncommutative algebra of such type which is generated by two elements is $K\left\langle x, y \mid x^{2}=y^{2}\right\rangle$ ([14). A related example on three generators that is not of this type is $K\left\langle x, y, z \mid x^{2}=y^{2}=z^{2}, z x=y z, z y=x z\right\rangle$. As an application of Theorem 2.4 one can show by elementary calculations that this algebra also is a prime Noetherian PI maximal order.

In the remainder of this section we discuss in full detail one more construction that illustrates Theorem 2.4 but also shows that certain assumptions in Theorem 1.1 are essential. Before this, we establish a useful general method for constructing nonabelian submonoids of abelian-by-finite groups that are maximal orders, starting from abelian maximal orders.

Proposition 3.1 Let $A$ be an abelian normal subgroup of finite index in a group $G$. Suppose that $B$ is a submonoid of $A$ so that $A=B B^{-1}$ and $B$ is a finitely generated maximal order. Let $S$ be a submonoid of $G$ such that $G=S S^{-1}$ and $S \cap A=B$. Then $S$ is a maximal order that satisfies the ascending chain condition on right ideals if and only if $S$ is maximal among all submonoids $T$ of $G$ with $T \cap A=B$.

Proof. First suppose $S$ is a maximal order that satisfies the ascending chain condition on right ideals. Suppose that $S \subseteq T \subseteq G$ for a submonoid $T$ of $G$ such that $S \cap A=B=T \cap A$. By assumption, $B$ is finitely generated. Since also $A$ is normal and of finite index in $G$, it thus follows (see the introduction) that $K[T]$ is Noetherian and it is a finitely generated right $K[B]$-module. Thus $T$ satisfies the ascending chain condition on one-sided ideals and $T=\bigcup_{i=1}^{n} t_{i} B$ for some $n \geq 1$ and $t_{i} \in T$. Since $G$ is finitely generated and abelian-by-finite, we know that for every $i$ there exists $z_{i} \in \mathrm{Z}(S)$ such that $z_{i} t_{i} \in S$. Let $z=z_{1} \cdots z_{k}$. Then $z t_{i} \in S$ and therefore $z T=\bigcup_{i} z t_{i}(S \cap A) \subseteq S$. Since $S$ is a maximal order, this implies that $T=S$. So, we have shown that $S$ is maximal among all submonoids $T \subseteq S S^{-1}$ such that $T \cap A=S \cap A$.

Conversely, assume that $S \subseteq G$ is maximal among all submonoids $T$ of $G$ with $T \cap A=B$. As above, because $S \cap A$ is finitely generated, $S$ satisfies the ascending chain condition on right ideals. Suppose that $T \subseteq G$ is a submonoid such that $S \subseteq T$ and $g T h \subseteq S$ for some $g, h \in G$. There exist $s, t \in S$ such that $s g, h t \in \mathrm{Z}(G) \cap B$. So $s g T h t \subseteq S$ and therefore $T z \subseteq S$ for some $z \in \mathrm{Z}(G) \cap B$. In particular $(T \cap A) z \subseteq S \cap A=B$. Since $S \cap A \subseteq T \cap A$ and $S \cap A$ is a maximal order, it follows that $T \cap A=S \cap A$. Because $S \subseteq T$, the assumption on $S$ then implies that $T=S$. Therefore $S$ is a maximal order.

In order to illustrate the above proposition with a concrete example, we start with the following construction of a monoid that contains the abelian monoid generated by $a_{1}, a_{2}, a_{3}, a_{4}$ and defined by the extra relation $a_{1} a_{2}=a_{3} a_{4}$. It was shown in 2] that the latter is a cancellative monoid that is a maximal order.

Example 3.2 The abelian monoid $B=\left\langle a_{1}, a_{2}, a_{3}, a_{4}, a_{5}, a_{6}\right\rangle$ defined by the relations $a_{1} a_{2}=a_{3} a_{4}=a_{5} a_{6}$ is a cancellative monoid that is a maximal order (in its torsion free group of quotients). 
Proof. Let $F=\left\langle x_{1}, \ldots, x_{8}\right\rangle$ be a free abelian monoid of rank 8 . Define

$$
\begin{aligned}
& b_{1}=x_{1} x_{2} x_{3} x_{4}, b_{2}=x_{5} x_{6} x_{7} x_{8}, b_{3}=x_{1} x_{2} x_{5} x_{6}, \\
& b_{4}=x_{3} x_{4} x_{7} x_{8}, b_{5}=x_{1} x_{3} x_{5} x_{7}, b_{6}=x_{2} x_{4} x_{6} x_{8} .
\end{aligned}
$$

Clearly, these $b_{i}$ satisfy the defining relations for $B$. We claim that actually $B \cong\left\langle b_{1}, \ldots, b_{6}\right\rangle$ under the map determined by $a_{i} \mapsto b_{i}, i=1, \ldots, 6$. In order to prove this, suppose that there is a relation $w=v$, where $w, v$ are nontrivial words in $b_{i}$. We need to show that this relation follows from $b_{1} b_{2}=b_{3} b_{4}=b_{5} b_{6}$. Cancelling in $F$, if needed, we may assume that each $b_{i}$ appears at most on one side of the relation. We also may assume that not both sides are divisible in $\left\langle b_{1}, \ldots, b_{6}\right\rangle$ by one of the equal words $b_{1} b_{2}, b_{3} b_{4}$ and $b_{5} b_{6}$. Further, on both sides of $v=w$ we need some $b_{i}$ with an even $i$. Indeed, suppose the contrary, then $x_{8}$ is not involved in $v$ and $w$. Hence also $b_{5}$ cannot occur because of $x_{7}$. But, as $b_{1}$ and $b_{3}$ generate a free abelian monoid of rank 2 , it then follows that $v$ and $w$ are identical words in the $b_{i}$ 's, as desired. Hence, by symmetry we may assume that $w$ contains $b_{2}^{i_{2}}$ with $i_{2}>0$ and $w$ does not contain $b_{4}$ nor $b_{6}$ as a factor, and $v$ does not contain $b_{2}$ as a factor and contains $b_{4}^{i_{4}} b_{6}^{i_{6}}$ for some nonnegative $i_{4}, i_{6}$ with $i_{2}=i_{4}+i_{6}$ (the latter follows by taking into the account the degree of $x_{8}$ in the respective words). Looking at $x_{4}$ we then get that $b_{1}$ appears in $w$. If $i_{6}=0$ then (looking at $x_{6}$ ) we get that $b_{3}$ is in $v$, a contradiction because $b_{1} b_{2}=b_{3} b_{4}$ divides then both $v$ and $w$. So $i_{6}>0$. Also $v$ must contain $x_{1}, x_{5}$ and so $b_{3}$ or $b_{5}$ is in $v$. The latter is not possible because then $v$ and $w$ are divisible by $b_{1} b_{2}=b_{5} b_{6}$, a contradiction. Thus, $b_{3}$ occurs in $v$. Then we must have $i_{4}=0$ because otherwise $b_{1} b_{2}=b_{3} b_{4}$ divides $v$ and $w$. So $w=b_{1}^{i_{1}} b_{2}^{i_{2}} b_{5}^{i_{5}}=b_{3}^{i_{3}} b_{6}^{i_{6}}=v$ for some $i_{1}, i_{2}, i_{6}>0$ and $i_{3}, i_{5} \geq 0$. Then the exponents of $x_{7}$ show that $i_{2}=i_{5}=0$, a contradiction. The claim follows. Hence we may indeed identify $a_{i}$ with $b_{i}$ and $B$ with $\left\langle b_{1}, \ldots, b_{6}\right\rangle$.

Next, suppose that $w=a_{2}^{i_{2}} a_{3}^{i_{3}} a_{4}^{i_{4}} a_{5}^{i_{5}} \in F \cap \operatorname{gr}(B)$. This is equivalent to the conditions: $i_{3}+i_{5} \geq 0, i_{3} \geq 0, i_{4}+i_{5} \geq 0, i_{4} \geq 0, i_{2}+i_{3}+i_{5} \geq 0, i_{2}+i_{3} \geq$ $0, i_{2}+i_{4}+i_{5} \geq 0, i_{2}+i_{4} \geq 0$. Let $j=\min \left\{i_{3}, i_{4}\right\} \geq 0$. Then $j+i_{2}+i_{5} \geq 0$. We choose $s, t \geq 0$ so that $i_{2}+s, i_{5}+t \geq 0$ and $s+t=j$. Then $w=$ $a_{1}^{s} a_{2}^{i_{2}+s} a_{3}^{i_{3}-j} a_{4}^{i_{4}-j} a_{5}^{i_{5}+t} a_{6}^{t}$. It is thus clear that $w \in B$. So $B=F \cap \operatorname{gr}(B)$. Since $F$ is a maximal order it then easily follows that $B$ is a maximal order.

We conclude with the promised illustration of Theorem 2.4. This example also shows that Theorem 1.1 cannot be extended to prime ideals of height exceeding 1.

Example 3.3 Let $K$ be any field and let $R=K\left\langle x_{1}, x_{2}, x_{3}, x_{4}\right\rangle$ be the algebra defined by the following relations:

$$
\begin{gathered}
x_{1} x_{4}=x_{2} x_{3}, x_{1} x_{3}=x_{2} x_{4}, x_{3} x_{1}=x_{4} x_{2} \\
x_{3} x_{2}=x_{4} x_{1}, x_{1} x_{2}=x_{3} x_{4}, x_{2} x_{1}=x_{4} x_{3} .
\end{gathered}
$$

Clearly, $R=K[S]$ for the monoid $S$ defined by the same presentation. Then $S$ is cancellative (but the group $S S^{-1}$ is not torsion free) and $R$ is a prime 
Noetherian PI-algebra that is a maximal order. Furthermore, there exists a prime ideal $P$ of $S$ so that $K[P]$ is not a prime ideal of $K[S]$.

Proof. Notice that each of the permutations (12)(34), (13)(24) and (14)(23) determines an automorphism of $S$. First we list some equalities in $S$, namely all relations between the elements of length 3 . For brevity, we use the index $i$ in place of the generator $x_{i}$.

$$
\begin{array}{cll}
112=134=244, & 113=124=344, & 114=123=343=321=411 \\
221=243=133, & 224=213=433, & 223=214=434=412=322 \\
331=342=122, & 334=312=422, & 332=341=121=143=233 \\
442=431=211, & 443=421=311, & 441=432=212=234=144 \\
131=142=232=241, & 141=132=242=231 \\
313=423=414=324, & 323=314=424=413 .
\end{array}
$$

It follows easily that $A=\left\langle x_{1}^{2}, x_{2}^{2}, x_{3}^{2}, x_{4}^{2}\right\rangle$ is an abelian submonoid and it is normal, that is $x A=A x$ for every $x \in S$. Moreover $x_{1}^{2} x_{4}^{2}=x_{2}^{2} x_{3}^{2}$ because $x_{2} x_{2} x_{3} x_{3}=x_{2} x_{3} x_{3} x_{2}=x_{1} x_{4} x_{4} x_{1}=x_{4} x_{4} x_{1} x_{1}$.

Let $a_{1}=x_{1} x_{4}, a_{2}=x_{4} x_{1}, a_{3}=x_{1}^{2}, a_{4}=x_{4}^{2}, a_{5}=x_{2}^{2}, a_{6}=x_{3}^{2}$ and $B=$ $\left\langle a_{1}, a_{2}, a_{3}, a_{4}, a_{5}, a_{6}\right\rangle$. From the above equalities it follows that $B$ is abelian and $s B=B s$ for every $s \in S$.

Every element of $S$ that is a word of length 3 is either of the form $x y y$ or of the form $x x_{1} x_{4}, x x_{4} x_{1}$ for some $x$. It is also easy to see that every element of $S$ is of the form $z u_{1} w_{1} \cdots u_{r} w_{r}$ or $z u_{1} w_{1} \cdots u_{r} w_{r} u_{r+1}$ or $z c$, where $z \in A$ and $c \in S$ is an element of length at most 2 in the $x_{j}$ and either $u_{i} \in\left\{x_{1}, x_{2}\right\}, w_{i} \in\left\{x_{3}, x_{4}\right\}$ or $u_{i} \in\left\{x_{3}, x_{4}\right\}, w_{i} \in\left\{x_{1}, x_{2}\right\}$ (for all $i$ ). So we may assume that $c \in\left\{x_{1} x_{2}, x_{2} x_{1}\right\}$. Here $r$ is a non-negative integer. Using the relations listed above (especially $\left.x_{1} x_{4} x_{1}=x_{2} x_{4} x_{2}, x_{4} x_{1} x_{4}=x_{3} x_{1} x_{3}\right)$ it may be checked that the even powers of $x_{1} x_{4}=x_{2} x_{3}$ and $x_{2} x_{4}=x_{1} x_{3}$ are equal and the even powers of $x_{4} x_{1}=x_{3} x_{2}$ and $x_{3} x_{1}=x_{4} x_{2}$ are equal. Also $\left\{x_{4} x_{1}, x_{1} x_{4}\right\}\left\{x_{1} x_{2}, x_{2} x_{1}\right\} \subseteq A\left\{x_{1} x_{3}, x_{3} x_{1}\right\}$. It follows that possible forms of elements of $S$ are

$$
\begin{aligned}
& z\left(x_{1} x_{4}\right)^{i}, z\left(x_{1} x_{4}\right)^{i} x_{1}, z\left(x_{1} x_{4}\right)^{i} x_{2}, z\left(x_{1} x_{4}\right)^{i} x_{1} x_{3}, z x_{1} x_{2} \\
& z\left(x_{4} x_{1}\right)^{i}, z\left(x_{4} x_{1}\right)^{i} x_{4}, z\left(x_{4} x_{1}\right)^{i} x_{3}, z\left(x_{4} x_{1}\right)^{i} x_{3} x_{1}, z x_{2} x_{1},
\end{aligned}
$$

with $z \in A, i \geq 0$. This leads to

$$
S=B \cup B x_{1} \cup B x_{2} \cup B x_{3} \cup B x_{4} \cup B x_{1} x_{3} \cup B x_{3} x_{1} \cup B x_{1} x_{2} \cup B x_{2} x_{1} \text {. (4) }
$$

It follows that $K[S]$ is finite module over the finitely generated commutative algebra $K[B]$. Hence $K[S]$ is a Noetherian PI-algebra.

Let $C$ be the free abelian group of rank 4 generated by elements $a, b, c, d$. Let $X$ be the free monoid on $x_{1}, x_{2}, x_{3}, x_{4}$. Consider the monoid homomorphism 
$\phi: X \longrightarrow M_{4}(K[C])$ defined by

$$
\begin{aligned}
x_{1} \mapsto\left(\begin{array}{cccc}
0 & a & 0 & 0 \\
1 & 0 & 0 & 0 \\
0 & 0 & 0 & a^{-1} b c \\
0 & 0 & 1 & 0
\end{array}\right), & x_{2} \mapsto\left(\begin{array}{cccc}
0 & 0 & b & 0 \\
0 & 0 & 0 & a^{-1} b c \\
1 & 0 & 0 & 0 \\
0 & a b^{-1} & 0 & 0
\end{array}\right), \\
x_{3} \mapsto\left(\begin{array}{cccc}
0 & 0 & b c d^{-1} & 0 \\
0 & 0 & 0 & a^{-1} b d \\
b^{-1} d & 0 & 0 & 0 \\
0 & a d^{-1} & 0 & 0
\end{array}\right), & x_{4} \mapsto\left(\begin{array}{cccc}
0 & b c d^{-1} & 0 & 0 \\
a^{-1} d & 0 & 0 & 0 \\
0 & 0 & 0 & d \\
0 & 0 & a d^{-1} & 0
\end{array}\right) .
\end{aligned}
$$

It is easy to check that these matrices satisfy the defining relations of $S$, so $\phi$ can be viewed as a homomorphism from $S$ to the group of monomial matrices over $C$. Moreover

$$
\begin{aligned}
a_{3} \mapsto\left(\begin{array}{cccc}
a & 0 & 0 & 0 \\
0 & a & 0 & 0 \\
0 & 0 & a^{-1} b c & 0 \\
0 & 0 & 0 & a^{-1} b c
\end{array}\right), & a_{5} \mapsto\left(\begin{array}{cccc}
b & 0 & 0 & 0 \\
0 & c & 0 & 0 \\
0 & 0 & b & 0 \\
0 & 0 & 0 & c
\end{array}\right) \\
a_{4} \mapsto\left(\begin{array}{cccc}
a^{-1} b c & 0 & 0 & 0 \\
0 & a^{-1} b c & 0 & 0 \\
0 & 0 & a & 0 \\
0 & 0 & 0 & a
\end{array}\right), & a_{6} \mapsto\left(\begin{array}{cccc}
c & 0 & 0 & 0 \\
0 & b & 0 & 0 \\
0 & 0 & c & 0 \\
0 & 0 & 0 & b
\end{array}\right), \\
a_{1} \mapsto\left(\begin{array}{cccc}
d & 0 & 0 & 0 \\
0 & b c d^{-1} & 0 & 0 \\
0 & 0 & b c d^{-1} & 0 \\
0 & 0 & 0 & d
\end{array}\right), & a_{2} \mapsto\left(\begin{array}{cccc}
b c d^{-1} & 0 & 0 & 0 \\
0 & d & 0 & 0 \\
0 & 0 & d & 0 \\
0 & 0 & 0 & b c d^{-1}
\end{array}\right) .
\end{aligned}
$$

The projection of the group $C^{\prime}$ generated by $\phi\left(a_{1}\right), \phi\left(a_{3}\right), \phi\left(a_{5}\right), \phi\left(a_{6}\right)$ onto the $(1,1)$-entry contains $a, b, c, d$, whence it is free abelian of rank 4 . So $C^{\prime} \cong C$. In particular, $\phi$ is injective on $B_{0}=\left\langle a_{1}, a_{3}, a_{5}, a_{6}\right\rangle$, and thus $B_{0}$ is a free abelian monoid of rank 4 . Let $B^{\prime}$ be the abelian monoid with presentation $\left\langle y_{1}, y_{2}, y_{3}, y_{4}, y_{5}, y_{6} \mid y_{1} y_{2}=y_{3} y_{4}=y_{5} y_{6}\right\rangle$. Clearly, we have natural homomorphisms $B^{\prime} \longrightarrow B \longrightarrow \phi(B)$. We also know that $\phi(B)$ generates a free abelian group of rank $\geq 4$ and $B^{\prime}$ has a group of quotients that is free abelian of rank 4 . So $B^{\prime}$ and $\phi(B)$ must be isomorphic, since otherwise under the map $B^{\prime} \longrightarrow \phi(B)$ we have to factor out an additional relation and the rank would decrease. It follows that $\phi$ is injective on $B$ and thus, because of Example 3.2. $B$ is a cancellative maximal order with group of quotients $N=B B^{-1} \cong \mathbb{Z}^{4}$. Note that $A A^{-1}=\operatorname{gr}\left(a_{3}, a_{5}, a_{6}\right)$. Using the defining relations $a_{1} a_{2}=a_{3} a_{4}=a_{5} a_{6}$ for $B$, it is readily verified that if $i, j, k \in \mathbb{Z}$ then $a_{3}^{i} a_{5}^{j} a_{6}^{k} \in A=\left\langle a_{3}, a_{4}, a_{5}, a_{6}\right\rangle$ if and only if $j, k \geq 0$ and $\min (j, k)+i \geq 0$.

The images under $\phi$ of the first 4 types listed in (2) have different patterns of nonzero entries in $M_{4}(K[C])$. The same applies to the first 4 types in (3). Notice that $\phi$ is injective on each of the 10 types. Suppose that $s=$ 
$z\left(x_{1} x_{4}\right)^{i} x_{1}, t=z^{\prime}\left(x_{4} x_{1}\right)^{j} x_{4}$ have the same image. Then $s x_{4}=z\left(x_{1} x_{4}\right)^{i+1}$ and $t x_{4}=z^{\prime}\left(x_{4} x_{1}\right)^{j} x_{4}^{2}$ have equal images. This is not possible because $\left(x_{1} x_{4}\right)^{i+1} A \cap$ $\left(x_{4} x_{1}\right)^{j} A=\emptyset$ by the above description of the group $B B^{-1} \cong \phi(B) \phi(B)^{-1}$. Similarly one deals with elements of any two different types listed in (2) and (3), showing that only elements the form $s=z x_{1} x_{2}, t=z^{\prime} x_{2} x_{1}$, where $z, z^{\prime} \in$ $A$, can have equal images. Then $\phi\left(z x_{1} x_{1} x_{4} x_{1}\right)=\phi\left(t x_{4} x_{2}\right)=\phi\left(s x_{4} x_{2}\right)=$ $\phi\left(z^{\prime} x_{4} x_{1} x_{2} x_{2}\right)$ and cancellativity of $\phi(B) \cong B$ yields $z x_{1} x_{1}=z^{\prime} x_{2} x_{2}$. Write $z=a_{3}^{i} a_{5}^{j} a_{6}^{k}$, with $i, j, k \in \mathbb{Z}$. Since $z, z^{\prime} \in A$, the above yields that $z^{\prime}=$ $a_{3}^{i+1} a_{5}^{j-1} a_{6}^{k}$ and $j, k \geq 0,-i$ and $j-1, k \geq 0,-(i+1)$. Notice that $a_{3} x_{2} x_{1}=$ $x_{1} x_{1} x_{2} x_{1}=x_{2} x_{2} x_{1} x_{2}=a_{5} x_{1} x_{2}$. Therefore, if $j-1 \geq-i$ then $a_{3}^{i} a_{5}^{j-1} a_{6}^{k} \in A$ and hence $z x_{1} x_{2}=a_{3}^{i} a_{5}^{j-1} a_{6}^{k} a_{5} x_{1} x_{2}=a_{3}^{i} a_{5}^{j-1} a_{6}^{k} a_{3} x_{2} x_{1}=z^{\prime} x_{2} x_{1}$. On the other hand, if $j=-i$ then $a_{4}^{j-1} a_{6}^{k-j} \in A$ and $a_{4} x_{1} x_{2}=a_{6} x_{2} x_{1}$. Hence we also get $z x_{1} x_{2}=a_{4}^{j} a_{6}^{k-j} x_{1} x_{2}=a_{4}^{j-1} a_{6}^{k-j} a_{4} x_{1} x_{2}=a_{4}^{j-1} a_{6}^{k-j} a_{6} x_{2} x_{1}=z^{\prime} x_{2} x_{1}$.

It follows that $\phi$ is injective on all elements of types (2),(3). Therefore $\phi$ is an embedding and thus $S$ is cancellative.

We identify $S$ with $\phi(S)$. Put $G=S S^{-1}$. Then $G=N \cup x_{1} N \cup x_{2} N \cup x_{1} x_{2} N$. Moreover $N \cong \mathbb{Z}^{4}$ and $N$ is a normal subgroup with $G / N$ the four group. From (4) it follows that $S \cap N=B$.

We now show that $G$ is dihedral free. To prove this, notice that $S$ acts by conjugation on $B$ and the generators $x_{i}$ of $S$ correspond to the following permutations $\sigma_{i}$ of the generating set of $B$ (the numbers $1,2,3,4,5,6$ correspond to the generators $\left.a_{1}, a_{2}, a_{3}, a_{4}, a_{5}, a_{6}\right)$.

$$
\sigma_{1}=(12)(56), \sigma_{2}=(12)(34), \sigma_{3}=(12)(34), \sigma_{4}=(12)(56) .
$$

Suppose $D \subseteq G$ is an infinite dihedral group such that the normalizer $N_{G}(D)$ of $D$ in $G$ is of finite index. Let $t \in D$ be an element of order 2. Then there exists $k \geq 1$ such that $a_{i}^{k} t a_{i}^{-k} t \in D$ for $i=1,2, \ldots, 6$. Clearly

$$
a_{i}^{k} t a_{i}^{-k} t=a_{i}^{k} a_{\sigma(i)}^{k} t^{2}=a_{i}^{k} a_{\sigma(i)}^{k},
$$

where $\sigma$ is the automorphism of $N$ determined by $t$. Since $t \in N x_{1} \cup N x_{2} \cup$ $N x_{1} x_{2}$, it follows that $\sigma$ is determined by the conjugation by $x_{1}, x_{2}$ or $x_{1} x_{2}$. So $\sigma$ permutes exactly two of the pairs $a_{1}, a_{2} ; a_{3}, a_{4}$ and $a_{5}, a_{6}$. It follows that $a_{i}^{k} a_{j}^{k}, a_{p}^{k} a_{q}^{k} \in D$ for two different pairs $i, j$ and $p, q$. Hence $\operatorname{rk}(N \cap D) \geq 2$, a contradiction. Therefore $G$ indeed is a dihedral free group.

Next we show that $\Delta^{+}(G)$ is trivial. For this, let $F$ be a finite normal subgroup of $G$. Since $N$ is torsion free, it is clear that $F$ is isomorphic with a subgroup of $\mathbb{Z}_{2} \times \mathbb{Z}_{2}$. A nontrivial element $t \in F$ must be of order 2 , whence as above we get that $a_{i}^{n} a_{j}^{n} t \in F$ for some $i \neq j$ and every $n \geq 1$. Therefore $F$ is infinite, a contradiction. It follows that $\Delta^{+}(G)$ is trivial. Therefore $K[G]$ is prime and hence $K[S]$ also is prime.

Note that in $G$ we have $x_{2}^{-1} x_{1}=x_{3} x_{4}^{-1}=x_{4} x_{3}^{-1}$ and $x_{1} x_{2}^{-1}=x_{3}^{-1} x_{4}=$ $x_{4}^{-1} x_{3}$. So $x_{2}^{-1} x_{1}$ is an element of order 2 .

We describe the minimal prime ideals of $S$. For this we first notice that it 
is easy to see that the minimal prime ideals of $B$ are:

$$
\begin{aligned}
Q=Q_{1} & =\left(a_{1}, a_{3}, a_{5}\right), & & Q_{2}=Q^{x_{1}}=\left(a_{2}, a_{3}, a_{6}\right), \\
Q_{3}=Q^{x_{2}} & =\left(a_{2}, a_{4}, a_{5}\right), & & Q_{4}=Q^{x_{1} x_{2}}=\left(a_{1}, a_{4}, a_{6}\right), \\
Q^{\prime}=Q_{5} & =\left(a_{2}, a_{3}, a_{5}\right), & & Q_{6}=\left(Q^{\prime}\right)^{x_{1}}=\left(a_{1}, a_{3}, a_{6}\right), \\
Q_{7}=\left(Q^{\prime}\right)^{x_{2}} & =\left(a_{1}, a_{4}, a_{5}\right), & & Q_{8}=\left(Q^{\prime}\right)^{x_{1} x_{2}}=\left(a_{2}, a_{4}, a_{6}\right) .
\end{aligned}
$$

Because of (5), it is easily verified that $a_{1} a_{2}$ is a central element of $S$ and that every ideal of $S$ contains a positive power of $a_{1} a_{2}$. In particular, $a_{1} a_{2}$ belongs to every prime ideal of $S$. Consider in $B$ the following $G$-invariant ideals:

$$
M=\left(a_{1} a_{3} a_{5}, a_{2} a_{3} a_{6}, a_{2} a_{4} a_{5}, a_{1} a_{4} a_{6}, a_{1} a_{2}\right)
$$

and

$$
M^{\prime}=\left(a_{2} a_{3} a_{5}, a_{1} a_{4} a_{5}, a_{1} a_{3} a_{6}, a_{2} a_{4} a_{6}, a_{1} a_{2}\right) .
$$

Again because of (5), it is easy to see that $b S b^{\prime} \subseteq a_{1} a_{2} S$ for every defining generator $b$ of $M$ and $b^{\prime}$ of $M^{\prime}$. It follows that a prime ideal of $S$ contains $M$ or $M^{\prime}$.

Notice that $x a_{1} a_{3} a_{5} y \in x y\left\{a_{1} a_{3} a_{5}, a_{2} a_{4} a_{5}, a_{2} a_{3} a_{6}, a_{1} a_{4} a_{6}\right\}$ for every $x, y \in$ $S$. Therefore $S a_{1} a_{3} a_{5} S \cap\left\langle a_{2}, a_{3}, a_{5}\right\rangle=\emptyset$ (for example, $x y a_{1} a_{3} a_{5} \notin\left\langle a_{2}, a_{3}, a_{5}\right\rangle$ because otherwise $x y=a_{1}^{-1} a_{3}^{-1} a_{5}^{-1}\left\langle a_{2}, a_{3}, a_{5}\right\rangle \cap(S \cap N)$, which is not possible because $S \cap N=B$ and $N=\operatorname{gr}\left(a_{1}, a_{2}, a_{3}, a_{5}\right)$ is free abelian of rank 4$)$. So there exists a (unique) ideal $P$ of $S$ that is maximal with respect to the property $P \cap\left\langle a_{2}, a_{3}, a_{5}\right\rangle=\emptyset$. It is easy to see that $P$ is a prime ideal of $S$. Since $a_{2} a_{3} a_{5} \notin P$, we get that $M^{\prime} \nsubseteq P$, whence $M \subseteq P$.

Let $E=a_{2} a_{3} a_{5}\left\langle a_{2}, a_{3}, a_{5}\right\rangle$. Then

$$
x_{4}\left\langle a_{1}, a_{3}, a_{6}\right\rangle x_{1} \subseteq E, x_{2}\left\langle a_{1}, a_{4}, a_{5}\right\rangle x_{2} \subseteq E, x_{1} x_{3}\left\langle a_{2}, a_{4}, a_{6}\right\rangle x_{3} x_{1} \subseteq E .
$$

Therefore $P \cap\left\langle a_{1}, a_{3}, a_{6}\right\rangle=\emptyset, P \cap\left\langle a_{1}, a_{4}, a_{5}\right\rangle=\emptyset, P \cap\left\langle a_{2}, a_{4}, a_{6}\right\rangle=\emptyset$. For every element $b \in B \backslash M$ the ideal $b B$ intersects one of the sets

$$
a_{2} a_{3} a_{5}\left\langle a_{2}, a_{3}, a_{5}\right\rangle, a_{1} a_{3} a_{6}\left\langle a_{1}, a_{3}, a_{6}\right\rangle, a_{1} a_{4} a_{5}\left\langle a_{1}, a_{4}, a_{5}\right\rangle, a_{2} a_{4} a_{6}\left\langle a_{2}, a_{4}, a_{6}\right\rangle .
$$

Since these sets do not intersect $P$, we get that $P \cap B=M$. Hence it is $G$ invariant and, because $M \subseteq Q_{1} \cap Q_{2} \cap Q_{3} \cap Q_{4}$ and all $Q_{i}$ are minimal primes of $B$, Corollary 1.3 yields that $P \cap B=M=Q_{1} \cap Q_{2} \cap Q_{3} \cap Q_{4}=\mathcal{B}(S M S)$ and $P$ is a minimal prime ideal of $S$. A similar argument shows that there exists an ideal $P^{\prime}$ of $\mathrm{S}$ that is maximal with respect to the property $P^{\prime} \cap\left\langle a_{1}, a_{3}, a_{5}\right\rangle=\emptyset$, and it follows that $P^{\prime} \cap B=M^{\prime}=Q_{5} \cap Q_{6} \cap Q_{7} \cap Q_{8}=\mathcal{B}\left(S M^{\prime} S\right)$ is the only other minimal prime of $S$.

In order to continue the proof we first show the following claim on the representation of elements of $B$.

Claim: Presentation

An element $b=a_{1}^{\alpha_{1}} a_{3}^{\alpha_{3}} a_{4}^{\alpha_{4}} a_{6}^{\alpha_{6}}$ of $N=B B^{-1}$ is in $B$ (with each $\alpha_{i} \in \mathbb{Z}$ ) if and only if $\alpha_{3}, \alpha_{4} \geq 0$ and either (i) $\alpha_{1} \geq 0$ and $\min \left(\alpha_{3}, \alpha_{4}\right)+\alpha_{6} \geq 0$ or (ii) 
$\alpha_{1}<0, \alpha_{3}+\alpha_{1}, \alpha_{4}+\alpha_{1} \geq 0$ and $\min \left(\alpha_{3}, \alpha_{4}\right)+\alpha_{1}+\alpha_{6} \geq 0$, or equivalently, $\min \left(\alpha_{3}+\alpha_{1}, \alpha_{4}+\alpha_{1}\right) \geq \max \left(0,-\alpha_{6}\right)$.

That the first condition is sufficient is easily verified. For the second condition, we rewrite $a_{1}^{\alpha_{1}}$ as $a_{3}^{\alpha_{1}} a_{4}^{\alpha_{1}} a_{2}^{-\alpha_{1}}$ and the result follows because of the first part (by interchanging $a_{1}$ with $a_{2}$ ).

To prove that they are necessary, suppose $b=a_{1}^{\alpha_{1}} a_{3}^{\alpha_{3}} a_{4}^{\alpha_{4}} a_{6}^{\alpha_{6}} \in B$, with each $\alpha_{i} \in \mathbb{Z}$. Because $B a_{4} \cap\left\langle a_{1}, a_{3}, a_{6}\right\rangle=\emptyset$ and $B a_{3} \cap\left\langle a_{1}, a_{4}, a_{6}\right\rangle=\emptyset$, it follows that $\alpha_{3}, \alpha_{4} \geq 0$. Suppose now that $\alpha_{1} \geq 0$. Then

$$
b=a_{1}^{\alpha_{1}} a_{3}^{\alpha_{3}-\min \left(\alpha_{3}, \alpha_{4}\right)} a_{4}^{\alpha_{4}-\min \left(\alpha_{3}, \alpha_{4}\right)} a_{5}^{\min \left(\alpha_{3}, \alpha_{4}\right)} a_{6}^{\alpha_{6}+\min \left(\alpha_{3}, \alpha_{4}\right)}
$$

and thus $a_{1}^{\alpha_{1}} a_{3}^{\alpha_{3}-\min \left(\alpha_{3}, \alpha_{4}\right)} a_{4}^{\alpha_{4}-\min \left(\alpha_{3}, \alpha_{4}\right)} a_{5}^{\min \left(\alpha_{3}, \alpha_{4}\right)} \in B a_{6}^{-\left(\alpha_{6}+\min \left(\alpha_{3}, \alpha_{4}\right)\right)}$. Since the exponent of $a_{3}$ or $a_{4}$ is 0 , this implies that $\alpha_{6}+\min \left(\alpha_{3}, \alpha_{4}\right) \geq 0$, as desired.

On the other hand, suppose that $\alpha_{1}<0$. Then $b=a_{2}^{-\alpha_{1}} a_{3}^{\alpha_{3}+\alpha_{1}} a_{4}^{\alpha_{4}+\alpha_{1}} a_{6}^{\alpha_{6}}$. The previous case yields that $\alpha_{3}+\alpha_{1} \geq 0, \alpha_{4}+\alpha_{1} \geq 0$ and $\min \left(\alpha_{3}+\alpha_{1}, \alpha_{4}+\right.$ $\left.\alpha_{1}\right)+\alpha_{6} \geq 0$, again as desired. This proves the claim Presentation.

Next we show that $S$ is a maximal order. First note that $B$ is a maximal order by Example 3.2. So, because of Proposition 3.1, it is sufficient to prove that if $s \in S S^{-1} \backslash S$ then $\langle S, s\rangle \cap N$ strictly contains $B$.

We know that $G=S S^{-1}=N \cup x_{1} N \cup x_{2} N \cup x_{1} x_{2} N$. If $s \in N \backslash B$ then clearly $\langle S, s\rangle \cap N$ strictly contains $B$. So, there are three cases to be dealt with: (1) $s=b x_{1} \in G \backslash S$, (2) $s=b x_{2} \in G \backslash S$, and (3) $s=b x_{1} x_{2} \in G \backslash S$, where $b \in N \backslash B$.

Case (1): $s=b x_{1} \in G \backslash S$. Obviously, $b x_{1} x_{4}=b a_{1} \in\langle S, s\rangle$. If $b a_{1} \in N \backslash B$, then we are done. So suppose $b a_{1} \in B$ and $b \in N \backslash B$. We write $b$ in the following form:

$$
a_{1}^{\alpha_{1}} a_{3}^{\alpha_{3}} a_{4}^{\alpha_{4}} a_{6}^{\alpha_{6}} .
$$

First, suppose that $\alpha_{1} \geq 0$. By the claim Presentation, from $b a_{1} \in B$ we get that $\min \left(\alpha_{3}, \alpha_{4}\right) \geq \max \left(-\alpha_{6}, 0\right)$. But, also from $b \in N \backslash B$ we get that $\min \left(\alpha_{3}, \alpha_{4}\right)<\max \left(-\alpha_{6}, 0\right)$, a contradiction. So, $\alpha_{1}$ has to be strictly negative.

Therefore, assume that $\alpha_{1}<0$. Then

$$
b=a_{1}^{\alpha_{1}} a_{3}^{\alpha_{3}} a_{4}^{\alpha_{4}} a_{6}^{\alpha_{6}}=a_{2}^{-\alpha_{1}} a_{3}^{\alpha_{3}+\alpha_{1}} a_{4}^{\alpha_{3}+\alpha_{1}} a_{6}^{\alpha_{6}}
$$

and

$$
b a_{1}=a_{2}^{-\alpha_{1}-1} a_{3}^{\alpha_{3}+\alpha_{1}+1} a_{4}^{\alpha_{3}+\alpha_{1}+1} a_{6}^{\alpha_{6}} .
$$

By the claim Presentation (by interchanging $a_{1}$ and $\left.a_{2}\right)$, we get that $\min \left(\alpha_{3}+\right.$ $\left.\alpha_{1}+1, \alpha_{4}+\alpha_{1}+1\right) \geq \max \left(-\alpha_{6}, 0\right)$, but also $\min \left(\alpha_{3}+\alpha_{1}, \alpha_{4}+\alpha_{1}\right)<\max \left(-\alpha_{6}, 0\right)$. Hence, $\min \left(\alpha_{3}+\alpha_{1}+1, \alpha_{4}+\alpha_{1}+1\right)=\max \left(-\alpha_{6}, 0\right)$. Suppose $\alpha_{6} \geq 0$. Then $\min \left(\alpha_{3}+\alpha_{1}+1, \alpha_{4}+\alpha_{1}+1\right)=0$. Therefore, $b=a_{1}^{\alpha_{1}} a_{3}^{\alpha_{3}} a_{4}^{\alpha_{4}} a_{6}^{\alpha_{6}}=$ $a_{1}^{-1} a_{2}^{\alpha_{3}} a_{4}^{\alpha_{4}-\alpha_{3}} a_{6}^{\alpha_{6}}$ and $\alpha_{4}>\alpha_{3}$ or $b=a_{1}^{-1} a_{2}^{\alpha_{4}} a_{3}^{\alpha_{3}-\alpha_{4}} a_{6}^{\alpha_{6}}$ and $\alpha_{3} \geq \alpha_{4}$. In the first case we get that $b x_{1} \in S$, since $x_{4}^{-1} x_{1}^{-1} x_{4} x_{4} x_{1}=x_{4}$. So this case gives a contradiction and is hence impossible. In the second case, $b x_{1} x_{1}=$ $a_{1}^{-1} a_{2}^{\alpha_{4}} a_{3}^{\alpha_{3}-\alpha_{4}+1} a_{6}^{\alpha_{6}} \in(\langle S, s\rangle \cap N) \backslash B$, as desired. If $\alpha_{6}<0, \min \left(\alpha_{3}+\alpha_{1}+\right.$ $\left.1, \alpha_{4}+\alpha_{1}+1\right)=-\alpha_{6}$ and therefore $b=a_{1}^{\alpha_{1}} a_{3}^{\alpha_{3}} a_{4}^{\alpha_{4}} a_{6}^{\alpha_{6}}=a_{1}^{-1} a_{2}^{-\alpha_{1}-1} a_{3}^{\alpha_{3}-\alpha_{4}} a_{5}^{-\alpha_{6}}$ 
if $\alpha_{4} \leq \alpha_{3}$ or $b=a_{1}^{-1} a_{2}^{-\alpha_{1}-1} a_{4}^{\alpha_{4}-\alpha_{3}} a_{5}^{-\alpha_{6}}$ if $\alpha_{3}<\alpha_{4}$. Since $-\alpha_{1}-1 \geq 0$ and $-\alpha_{6}>0$, by interchanging $a_{5}$ and $a_{6}$, this case is completely similar to the case where $\alpha_{6} \geq 0$. This finishes the proof of Case (1).

Case (2): $s=b x_{2} \in G \backslash S$. The permutation $\sigma=(12)(34)$ determines an automorphism $\sigma$ on $S$ with $\sigma(B)=B$. Clearly, $\sigma(s)=b^{\prime} x_{1}$ with $b^{\prime}=\sigma(b) \notin B$. From Case (1) we get that $\langle S, \sigma(s)\rangle \cap N$ properly contains $B$. Again applying $\sigma$ to the latter we get that $\langle S, s\rangle \cap N$ properly contains $B$, as required.

Case (3): $s=b x_{1} x_{2} \in G \backslash S$. Clearly, $b x_{1} x_{2} x_{2} x_{4}=b a_{1} a_{6} \in\langle S, s\rangle$. If $b a_{1} a_{6} \in N \backslash B$, then we are done. So suppose $b a_{1} a_{6} \in B$ and $b \in N \backslash B$. Write $b=a_{1}^{\alpha_{1}} a_{3}^{\alpha_{3}} a_{4}^{\alpha_{4}} a_{6}^{\alpha_{6}}$. We know that $\alpha_{3}, \alpha_{4} \geq 0$ and we consider again the two cases: $\alpha_{1} \geq 0$ and $\alpha_{1}<0$, separately.

First assume that $\alpha_{1} \geq 0$. Since $b a_{1} a_{6} \in B$ and $b \notin B$, we get that $\min \left(\alpha_{3}, \alpha_{4}\right)=-\alpha_{6}-1$. Then $b=a_{1}^{\alpha_{1}} a_{3}^{\alpha_{3}} a_{4}^{\alpha_{4}} a_{6}^{\alpha_{6}}=a_{1}^{\alpha_{1}} a_{3}^{\alpha_{3}-\alpha_{4}} a_{5}^{-\alpha_{6}-1} a_{6}^{-1}$, if $\alpha_{4} \leq \alpha_{3}$, or $b=a_{1}^{\alpha_{1}} a_{4}^{\alpha_{4}-\alpha_{3}} a_{5}^{-\alpha_{6}-1} a_{6}^{-1}$, if $\alpha_{3}<\alpha_{4}$. Now, let $\alpha_{1}>0$. Then $b x_{1} x_{2} \in S$, since $x_{1} x_{4} x_{3}^{-1} x_{3}^{-1} x_{1} x_{2}=x_{2} x_{4}$, a contradiction. So this case is impossible. Therefore, $\alpha_{1}=0$. If $\alpha_{3}<\alpha_{4}$, then $b x_{1} x_{2} \in S$, since $x_{4} x_{4} x_{3}^{-1} x_{3}^{-1} x_{3} x_{4}=x_{2} x_{1}$, a contradiction, so this case is again impossible. If $\alpha_{4} \leq \alpha_{3}$, then $b x_{1} x_{2} x_{3} x_{1}=b a_{2} a_{3} \in(\langle S, s\rangle \cap N) \backslash B$, as desired.

Finally, suppose $\alpha_{1}<0$. Then $b=a_{1}^{\alpha_{1}} a_{3}^{\alpha_{3}} a_{4}^{\alpha_{4}} a_{6}^{\alpha_{6}}=a_{2}^{-\alpha_{1}} a_{3}^{\alpha_{3}+\alpha_{1}} a_{4}^{\alpha_{4}+\alpha_{1}} a_{6}^{\alpha_{6}}$ and $b a_{1} a_{6}=a_{2}^{-\alpha_{1}-1} a_{3}^{\alpha_{3}+\alpha_{1}+1} a_{4}^{\alpha_{4}+\alpha_{1}+1} a_{6}^{\alpha_{6}+1}$. By the claim Presentation (by interchanging $a_{1}$ and $\left.a_{2}\right)$, we get that $\min \left(\alpha_{3}+\alpha_{1}+1, \alpha_{4}+\alpha_{1}+1\right) \geq \max \left(-\alpha_{6}-\right.$ $1,0)$, but also $\min \left(\alpha_{3}+\alpha_{1}, \alpha_{4}+\alpha_{1}\right)<\max \left(-\alpha_{6}, 0\right)$. If $\alpha_{6} \geq 0, \min \left(\alpha_{3}, \alpha_{4}\right)+$ $\alpha_{1}=-1$ and $b=a_{1}^{-1} a_{2}^{\alpha_{4}} a_{3}^{\alpha_{3}-\alpha_{4}} a_{6}^{\alpha_{6}}$, if $\alpha_{4} \leq \alpha_{3}$, or $b=a_{1}^{-1} a_{2}^{\alpha_{3}} a_{4}^{\alpha_{4}-\alpha_{3}} a_{6}^{\alpha_{6}}$, if $\alpha_{3}<$ $\alpha_{4}$. So, we have the same conditions here as in Case 1 and the result follows. If $\alpha_{6}<0$, there are two possibilities: $\min \left(\alpha_{3}, \alpha_{4}\right)+\alpha_{1}=-\alpha_{6}-1$ or $\min \left(\alpha_{3}, \alpha_{4}\right)+$ $\alpha_{1}=-\alpha_{6}-2$. In the first case, we get that $b=a_{1}^{-1} a_{2}^{-\alpha_{1}-1} a_{3}^{\alpha_{3}-\alpha_{4}} a_{5}^{-\alpha_{6}}$ if $\alpha_{4} \leq$ $\alpha_{3}$, or $b=a_{1}^{-1} a_{2}^{-\alpha_{1}-1} a_{4}^{\alpha_{4}-\alpha_{3}} a_{5}^{-\alpha_{6}}$ if $\alpha_{3}<\alpha_{4}$. Since $-\alpha_{1}-1 \geq 0$ and $-\alpha_{6}>0$, by interchanging $a_{5}$ and $a_{6}$, this case is completely similar to the case where $\alpha_{6} \geq 0$ and hence can also be treated as in Case 1. Finally, if the second possibility holds, that is $\min \left(\alpha_{3}, \alpha_{4}\right)+\alpha_{1}=-\alpha_{6}-2$, then $b=a_{1}^{-2} a_{2}^{-2-\alpha_{1}} a_{3}^{\alpha_{3}-\alpha_{4}} a_{5}^{-\alpha_{6}}$, if $\alpha_{4} \leq \alpha_{3}$, or $b=a_{1}^{-2} a_{2}^{-2-\alpha_{1}} a_{4}^{\alpha_{4}-\alpha_{3}} a_{5}^{-\alpha_{6}}$, if $\alpha_{4}>\alpha_{3}$, so we always get that $b a_{2} a_{3} \in(\langle S, s\rangle \cap N) \backslash B$, as desired.

This finishes the proof of the fact that $S$ is a maximal order. Since $P \cap B$ is invariant for every minimal prime $P$ of $S$, it then follows from Theorem 2.4 that $K[S]$ is a maximal order.

Let $V$ be the ideal of $S$ generated by the elements $a_{3}, a_{4}, a_{5}, a_{6}, x_{1} x_{2}, x_{2} x_{1}$. It easily follows that the elements of $S \backslash V$ are of the form

$$
\begin{gathered}
\left(x_{1} x_{4}\right)^{i},\left(x_{1} x_{4}\right)^{i} x_{1}, \quad\left(x_{1} x_{4}\right)^{i} x_{2}, \quad\left(x_{1} x_{4}\right)^{i} x_{1} x_{3}, \\
\left(x_{4} x_{1}\right)^{i}, \quad\left(x_{4} x_{1}\right)^{i} x_{4},\left(x_{4} x_{1}\right)^{i} x_{3}, \quad\left(x_{4} x_{1}\right)^{i} x_{3} x_{1},
\end{gathered}
$$

where $i$ is a non-negative integer. Then $S \backslash V=\{1\} \cup I$ where the set $I$ can be written in matrix format as a union of disjoint sets:

$$
\left(\begin{array}{ll}
I_{11} & I_{12} \\
I_{21} & I_{22}
\end{array}\right),
$$


with $I_{11}=\left\langle a_{2}\right\rangle a_{2} \cup\left\langle a_{2}\right\rangle x_{3} x_{1}, I_{12}=\left\langle a_{2}\right\rangle x_{3} \cup\left\langle a_{2}\right\rangle x_{4}, I_{21}=x_{1}\left\langle a_{2}\right\rangle \cup x_{2}\left\langle a_{2}\right\rangle$ and $I_{22}=\left\langle a_{1}\right\rangle a_{1} \cup\left\langle a_{1}\right\rangle x_{1} x_{3}$. Moreover, $I_{i j} I_{k l} \subseteq I_{i l}$ if $j=k$, and is contained in $V$ otherwise. In $S / V$ the set $I \cup\{0\}$ is an ideal and the semigroup $I_{11}$ (treated as a subsemigroup of $S$ ) has a group of quotients $H=\operatorname{gr}\left(a_{2}, x_{3} x_{1}\right)$. Since $a_{2}^{2}=\left(x_{3} x_{1}\right)^{2}$ and $a_{2}\left(x_{3} x_{1}\right)=\left(x_{3} x_{1}\right) a_{2}$, we get that $H$ is isomorphic with $\mathbb{Z} \times \mathbb{Z}_{2}$. From the matrix pattern of $I$ it follows that $S / V$ is a prime semigroup. So $V$ is a prime ideal of $S$. However, because $K[H]$ and thus $K\left[I_{11}\right]$ is not prime, standard generalized matrix ring arguments yield that $K[S] / K[V]$ is not prime.

\section{References}

[1] Anderson D.F., Graded Krull domains, Comm. Algebra 7 (1979), 79-106.

[2] Anderson D.F., The divisor class group of a semigroup ring, Comm. Algebra 8 (1980), 467-476.

[3] Brown K.A., Height one primes of polycyclic group rings, J. London Math. Soc. 32 (1985), 426-438.

[4] Brown K.A., Corrigendum and addendum to 'Height one primes of polycyclic group rings', J. London Math. Soc. 38 (1988), 421-422.

[5] Chamarie M., Anneaux de Krull non commutatifs, Thèse, Université Claude-Bernard - Lyon I, 1981.

[6] Chamarie M., Anneaux de Krull non commutatifs, J. Algebra 72 (1981), 210-222.

[7] Chatters A.W. and Jordan D.A., Non-commutative unique factorisation rings, J. London Math. Soc. (2) 33 (1986), 22-32.

[8] Chin W. and Quinn D., Rings graded by polycyclic-by-finite groups, Proc. Amer. Math. Soc. 102 (1988), 235-241.

[9] Chouinard II L.G., Krull semigroups and divisor class groups, Canad. J. Math. 23 (1981), 1459-1468.

[10] Cohen M. and Rowen L., Group graded rings, Comm. Algebra 11 (1983), $1253-1270$.

[11] Etingof P., Guralnick R. and Soloviev A., Indecomposable set-theoretical solutions to the quantum Yang-Baxter equation on a set with a prime number of elements, J. Algebra 249 (2001), 709-719.

[12] Etingof P., Schedler T. and Soloviev A., Set-theoretical solutions of the quantum Yang-Baxter equation, Duke Math. J. 100 (1999), 169-209.

[13] Fossum R., The Divisor Class Group of a Krull Domain, Springer-Verlag, New York, 1973.

[14] Gateva-Ivanova T. and Van den Bergh M., Semigroups of $I$-type, J. Algebra, 206 (1998), 97-112.

[15] Gateva-Ivanova T., A combinatorial approach to the set-theoretic solutions of the Yang-Baxter equation, J. Math. Phys. 45 (2004), 3828-3858. 
[16] Gilmer R., Commutative Semigroup Rings, Univ. Chicago Press, 1984.

[17] Goodearl K.R. and Warfield R.B., An Introduction to Noncommutative Noetherian Rings, Cambridge Univ. Press, New York, 1989.

[18] Goffa I. and Jespers E., Monoids of IG-type and maximal orders, J. Algebra 308 (2007), 44-62.

[19] Jespers E., Krempa J. and Puczylowski E.R., On radicals of graded rings, Comm. Algebra 10 (1982), 1849-1854.

[20] Jespers E. and Okniński J., Binomial semigroups, J. Algebra 202 (1998), 250-275.

[21] Jespers E. and Okniński J., Semigroup algebras and Noetherian maximal orders, J. Algebra 238 (2001), 590-622.

[22] Jespers E. and Okniński J., Submonoids of polycyclic-by-finite groups and their algebras, Algebras Repres. Theory 4 (2001), 133-153.

[23] Jespers E. and Okniński J., Noetherian semigroup algebras of submonoids of polycyclic-by-finite groups, Bull. London Math. Soc. 38 (2006), 421-428.

[24] Jespers E. and Okniński J., Noetherian Semigroup Algebras, Algebra and Applications vol.7, Springer, 2007.

[25] Jespers E. and Wang Q., Height one prime ideals in semigroup algebras satisfying a polynomial identity, J. Algebra 248 (2002), 118-131.

[26] Krause G.R. and Lenagan T.H., Growth of Algebras and Gelfand-Kirillov Dimension, Revised edition. Graduate Studies in Mathematics, 22. American Mathematical Society, Providence, RI, 2000.

[27] McConnell J.C. and Robson J.C., Noncommutative Noetherian Rings, Wiley, New York, 1987.

[28] Okniński J., Semigroup Algebras, Marcel Dekker, New York, 1991.

[29] Okniński J., In search for Noetherian algebras, in: Algebra - Representation Theory, NATO ASI, pp. 235-247, Kluwer, 2001.

[30] Okniński J., Prime ideals of cancellative semigoups, Comm. Algebra 32 (2004), 2733-2742.

[31] Passman D.S., The Algebraic Structure of Group Rings, Wiley, New York, 1977.

[32] Passman D.S., Infinite Crossed Products, Academic Press, New York, 1989.

[33] Rump W., A decomposition theorem for square-free unitary solutions of the quantum Yang-Baxter equation, Adv. Math. 193 (2005), 40-55.

[34] Wauters P., On some subsemigroups of noncommutative Krull rings, Comm. Algebra 12 (1984), 1751-1765.

I. Goffa and E. Jespers

Department of Mathematics

Vrije Universiteit Brussel

Pleinlaan 2

1050 Brussel, Belgium

efjesper@vub.ac.be and igoffa@vub.ac.be
J. Okniński

Institute of Mathematics

Warsaw University

Banacha 2

02-097 Warsaw, Poland

okninski@mimuw.edu.pl 